\title{
Single-cell evaluation reveals shifts in the tumor- immune niches that shape and maintain aggressive lesions in the breast
}

\section{Vidya C. Sinha}

The University of Texas MD Anderson Cancer Center https://orcid.org/0000-0002-3604-1168

Amanda L. Rinkenbaugh

The University of Texas MD Anderson Cancer Center https://orcid.org/0000-0003-3276-6863

Mingchu Xu

The University of Texas MD Anderson Cancer Center

\section{Xinhui Zhou}

The University of Texas MD Anderson Cancer Center

Xiaomei Zhang

The University of Texas MD Anderson Cancer Center

\section{Sabrina Jeter-Jones}

The University of Texas MD Anderson Cancer Center

Jiansu Shao

The University of Texas MD Anderson Cancer Center

\section{Yuan Qi}

The University of Texas MD Anderson Cancer Center

John A. Zebala

Syntrix Pharmaceuticals, Inc.

\section{Dean Y. Maeda}

Syntrix Pharmaceuticals, Inc.

\section{Florencia McAllister}

The University of Texas MD Anderson Cancer Center

Helen Piwnica-Worms ( $\square$ hpiwnica-worms@mdanderson.org )

The University of Texas MD Anderson Cancer Center

\section{Research Article}

Keywords: Cancer, breast, DCIS, mouse model, single-cell RNA sequencing, imaging mass cytometry, metaplasia, plasticity, neutrophils, IL-17

Posted Date: February 8th, 2021 
DOl: https://doi.org/10.21203/rs.3.rs-179498/v1

License: (c) (1) This work is licensed under a Creative Commons Attribution 4.0 International License. Read Full License 


\section{Abstract}

There is an unmet clinical need for stratification of breast lesions as indolent or aggressive to tailor treatment. Here, single-cell transcriptomics and multiparametric imaging applied to a mouse model of breast cancer revealed that the aggressive tumor niche is characterized by an expanded basal-like population, specialization of tumor subpopulations, and mixed-lineage tumor cells potentially serving as a transition state between luminal and basal phenotypes. Despite vast tumor cell-intrinsic differences, aggressive and indolent tumor cells were functionally indistinguishable once isolated from their local niche, suggesting a role for non-tumor collaborators in determining aggressiveness. Aggressive lesions harbored fewer total but more suppressed-like T cells, and elevated tumor-promoting neutrophils and IL17 signaling, disruption of which increased tumor latency and reduced the number of aggressive lesions. Our study provides insight into tumor-immune features distinguishing indolent from aggressive lesions, identifies heterogeneous populations comprising these lesions, and supports a role for IL-17 signaling in aggressive progression.

\section{Introduction}

Ductal carcinoma in situ (DCIS) is a non-invasive lesion of the breast that is thought to serve as a nonobligate precursor of invasive breast cancer. DCIS now comprises approximately $15-30 \%$ of newly diagnosed breast cancers in the United States (DeSantis et al., 2019; Lehman et al., 2017; Siegel et al., 2018). Currently, almost all DCIS patients are treated indiscriminately with surgical resection of the lesion, with or without adjuvant endocrine or radiation therapy, an approach unlikely to be optimal for most patients (Silverstein and Lagios, 2014). Unfortunately, it is currently not possible to differentiate lesions that will progress to invasive, potentially lethal disease from those that will remain indolent (and thus could be spared treatment), underscoring the clinical need for consensus stratification of breast lesions as indolent or aggressive to guide treatment. Indeed, several clinical studies are underway to address these urgent questions (Elshof et al., 2015; Francis et al., 2015; Han and Khan, 2018; Hwang et al., 2019; Hwang and Malek, 2019; Kanbayashi and Iwata, 2017; van Seijen et al., 2019).

Improvement in the clinical management of DCIS is limited by our incomplete understanding of early breast cancer biology. Studies on intraepithelial breast neoplasias have been limited by the technical challenges of isolating small lesions from adjacent normal tissue and obtaining sufficient tumor material for downstream analyses (Sinha and Piwnica-Worms, 2018). Nonetheless, valuable insight from these studies have cumulatively led to at least two major, non-mutually exclusive schools of thought to explain the differences between indolent and aggressive disease. One hypothesis focuses primarily on changes that occur intrinsically within transformed epithelial cells as they break through the basement membrane, while another emphasizes a major role for a distorted lesion niche that switches from inhibiting invasion to permitting (and even promoting) it (Allen et al., 2014; Campbell et al., 2017; Cowell et al., 2013; Emery et al., 2009; Gil Del Alcazar et al., 2017; Hu et al., 2008; Lee et al., 2012; Seth et al., 2003). 
Recently, studies have evaluated early stage breast lesions using single-cell approaches assaying both genotype (Casasent et al., 2018; Heselmeyer-Haddad et al., 2012) and phenotype (Gerdes et al., 2018), allowing insights into in situ lesion biology that may have been partly obscured by bulk analyses. However, a single-cell transcriptional view of intraepithelial breast lesions is currently lacking, and the heterogeneous transcriptional subpopulations that comprise indolent and aggressive lesions are undefined.

To identify features that distinguish indolent and aggressive lesions and to identify potential determinants of lesion aggressiveness, we used an ErbB2 mouse model of breast cancer that generates both indolent (in situ, slow growing) and aggressive (invasive, rapidly growing) lesions within the same mammary gland. We interrogated the tumor and immune niche compartments of both indolent and aggressive lesions, and functionally measured the degree to which these features shape lesion behavior, using a combination of single-cell transcriptomic analyses, multiparametric imaging, and functional studies. Our work provides insight on the heterogeneous transcriptional and phenotypic populations that comprise these lesions, supports a role for IL-17 signaling in mammary lesion progression, and generates a collection of tumor and immune niche features that may be useful in identifying lesions that are capable of aggressive behavior, including those that might be otherwise considered indolent.

\section{Results}

\section{Aggressive progression occurs in a minority of caErbB2-expressing breast lesions}

To model early stages of tumor initiation, we delivered lentiviral particles carrying constitutively-activated Erbb2 tagged with HA and GFP (caErbB2) through the lactiferous glands of adult virgin female FVB mice (Bu et al., 2009; Siwko et al., 2008). The resulting caErbB2-initiated lesions within the same mammary gland progressed asynchronously, as evidenced by the simultaneous presence of lesions at various stages of advancement (Fig $1 A$, B; Supp fig $1 A$ ). A minor population ( $5-10 \%$ ) of lesions grew extremely rapidly such that, beyond $\sim 1 \mathrm{~mm}^{2}$, these lesions were significantly larger (30-40 fold; $\left.p<0.0001\right)$ than the remaining $90-95 \%$ of lesions (Fig $1 C$ ). Based on this asynchronous progression, we inferred that the majority of lesions progressed at a relatively indolent pace and remained small, while a minority advanced aggressively to form large tumors.

Histopathological evaluation of lesion-bearing mammary glands confirmed that while the majority of indolent lesions remained constrained by an intact basement membrane, aggressive lesions consistently exhibited widespread invasion beyond the confines of the duct (Fig 1D, top panel; Supp fig 1B). Immunostaining of the basement membrane protein collagen IV not only confirmed a loss of basement membrane integrity in aggressive lesions, but also revealed extremely irregular and intra-tumoral expression of collagen IV. Although indolent and aggressive lesions both classified as Luminal B based on HER2, Ki67, and estrogen receptor (ER) expression (Goldhirsch et al., 2011) (Supp fig 1C-E), aggressive lesions often exhibited spindloid and squamous metaplastic tumor cells, with the latter frequently adjacent to keratin-like structures or keratin pearls (Supp fig 1A). Finally, we observed that indolent and 
aggressive lesions were each associated with distinct local microenvironments. Most notably, aggressive lesions were associated with a heavy immune infiltrate compared to indolent lesions (Fig 1D, bottom panel).

Despite lesions in the same animal experiencing the same initiating oncogene (active Erbb2), only a relatively rare subset of these lesions gained the functional capacity to progress aggressively or recruit immune cells. These observations strongly suggest that additional factors cooperated to determine the likelihood and rate of lesion progression, and that only a relatively rare subset of these lesions gained the functional capacity to progress aggressively. Thus, this model system provides a unique opportunity to distinguish lesions that progress rapidly from those that progress at a relatively indolent pace, and to identify potential cell intrinsic and extrinsic determinants of aggressive lesion progression.

\section{Tumor cells from indolent versus aggressive lesions are transcriptionally distinct}

To more thoroughly characterize cell-intrinsic differences between indolent and aggressive lesions, bulk RNA sequencing (RNA-seq) was performed on tumor cells isolated from indolent and aggressive lesions (Fig 2A, Supp fig 2A).

Transcriptional analysis of these tumor cells revealed that indolent and aggressive lesions were distinct from one another, as determined by principal component analysis (PCA) (Fig 2B), and differential gene expression (DE) analysis, which identified over 8000 differentially expressed genes (8209 DE at FDR $<0.05 ; 1360$ DE at FDR $<0.05$, fold change $>2 ;$ Fig 2C, Supp fig 2B, C). Gene set enrichment analysis (GSEA) showed significant differences in several key cancer-associated pathways (Fig 2D, E; Supp table 1, 2). In particular, aggressive lesions exhibited enrichment of cell cycle and DNA replication pathways (Fig $2 E$ ), as well as E2F- and Myc-associated Hallmark pathways (Fig 2D). Furthermore, genes associated with DNA repair, G2/M checkpoint, p53 signaling, and metabolic (oxidative phosphorylation, glycolysis) pathways were also increased (Fig 2D, E), indicative of elevated oncogenic, replicative, and metabolic stress known to accompany rapid tumor growth and progression (Hanahan and Weinberg, 2011). Aggressive lesions also overexpressed EMT pathway genes (Fig 2D), consistent with reports that invasiveness is facilitated by the transition of epithelial cells to a more mesenchymal state (Nieto et al., 2016; Ribatti et al., 2020; Yang et al., 2020). These findings also confirmed our own histopathological observations of spindloid and squamous metaplastic cells in aggressive lesions. Additionally, aggressive lesions exhibited negative enrichment of immune-associated pathways (Fig 2D) despite heavy immune infiltration into these lesions (Fig 1D, bottom panel), suggesting that aggressive tumor cells may be capable of dampening anti-tumor immunity.

Taken together, the transcriptional profile of large, rapidly progressing lesions is consistent with aggressive growth, activation of developmental pathways leading to epithelial metaplasia, and inhibition of anti-tumor immune responses that might have otherwise limited tumor growth and progression. 


\section{Cell-intrinsic features are insufficient to drive aggressive lesion behavior}

To ascertain the degree to which aggressive lesion behavior was determined by cell-intrinsic features, we tested the lesion-forming capacity of epithelial cells isolated from both indolent and aggressive lesions. We hypothesized that if lesion behavior was driven primarily by cell-intrinsic properties, then cells from indolent lesions would give rise to slow-growing, in situ lesions, while cells from aggressive lesions would give rise to rapidly-progressing, invasive lesions. To test this hypothesis, we isolated GFP+ tumor cells from indolent and aggressive lesions and immediately re-injected them into the mammary ducts of wildtype, non-tumor-bearing adult female mice to test their ability to give rise to new lesions. Surprisingly, we found that indolent and aggressive lesions were indistinguishable from one another in their ability to initiate new tumors when placed in matched microenvironments of new, non-tumor-bearing hosts (Fig $2 F$ ). In particular, we found that cells derived from indolent lesions were capable of forming both in situ and invasive lesions, as were those derived from aggressive lesions (Fig 2G). Additionally, injection of a limiting dilution of cells revealed no significant difference in lesion forming capacity of indolent versus aggressive lesions calculated using Extreme Limiting Dilution Analysis (Hu and Smyth, 2009) ( $p=0.34$, Fig $2 H)$. Taken together, these results suggest that cell-intrinsic features alone were insufficient to drive aggressive behavior.

\section{The aggressive tumor niche harbors an expanded basal-like population}

To investigate how co-evolving epithelial and immune cells might functionally govern lesion aggressiveness, we performed single-cell RNA sequencing (scRNA-seq) of tumor (GFP+) and immune (CD45+) cells isolated from both indolent and aggressive lesions (Fig 3A). Analysis of the tumor epithelial cell compartment revealed two luminal-like populations (major Luminal 1 and minor Luminal 2) that comprised the bulk of indolent lesions, and a basal-like population (Basal) that comprised the bulk of aggressive lesions (Fig 3B-F, Supp fig 3). We also identified contaminant populations of fibroblasts and immune cells within the GFP+ fraction (Supp fig 3c), that were useful for validating our clustering approach. Immunostaining by imaging mass cytometry (Fig 4) for luminal and basal cytokeratins (CK8 and CK5, respectively) confirmed that indolent and aggressive lesions were indeed comprised of two major distinct epithelial subtypes. Consistent with some types of human in situ lesions, indolent lesions in our model were comprised primarily of cuboidal-shaped luminal (CK8+) tumor cells, relatively uniform in appearance throughout the lesion cross-section, with a single discontinuous layer of basal (CK5+) cells in the surrounding basal compartment (Pinder, 2010; Yeh and Mies, 2008). In contrast, invasive lesions harbored an expanded $\mathrm{CK} 5+$ population that was not restricted to the basal compartment and instead intermixed with CK8+ cells within the tumor bulk (Fig 4A, B; Supp fig 4A, D).

Interestingly, single-cell analysis identified an intermediate cell population (designated LB, for LuminalBasal) whose transcriptional profile contained features of both Luminal 1 and Basal clusters, and which 
expanded as lesions advanced. A similar luminal-basal mixed-lineage population was also detected histologically within invasive lesions co-expressing luminal and basal epithelial markers (CK8, CK5; Fig $4 B$; Supp fig 4), a subset of which also expressed the mesenchymal marker vimentin (Fig 4C). These cells

could be identified among single positive cells (Supp fig $4 A, B$ ). Very rarely, cells that appeared to be in the process of transitioning between single and double CK positive states were observed (Supp fig $4 C$ ). The emergence of this mixed-lineage population, in addition to the broad presence of tumor cell metaplasia within the aggressive niche, suggests that rapid lesion progression in this model may be associated with loss of epithelial identity, increased plasticity, and/or expansion of precursor populations (Mani et al., 2008).

To visualize the degree of transcriptional relatedness between tumor cells, we constructed pseudotemporal trajectories by Monocle v2 (Qiu et al., 2017b; Trapnell et al., 2014). As suggested by our clustering analysis, we found a transcriptional continuum between Luminal 1 cells and Basal cells, through LB intermediates (Fig 3G, H). In contrast, Luminal 2 cells were found to be relatively transcriptionally distinct from other epithelial populations (Fig $3 \mathrm{H}$, left panel). Taken together these data suggest that some breast cancer cells may undergo a cell-state transition between luminal-like and basallike phenotypes, through an identifiable intermediate transition state, during progression from in situ to invasive disease.

\section{Tumor subpopulations exhibit heterogeneous pathway enrichment that become more pronounced in aggressive lesions}

Given the shift in frequencies of different tumor cell types in aggressive and indolent lesions, we next asked whether these different tumor cell populations exhibited detectable differences, beyond cell identity, in their imputed function. Evaluation of Hallmark and KEGG gene signatures by gene set variation analysis (GSVA, (Fig 5A, B) largely aligned with differences identified by bulk transcriptomic profiling of indolent and aggressive tumor cells. The ErbB2 signaling pathway (KEGG) was enriched within the Luminal populations, which was expected given the oncogenic event introduced into these cells was caErbB2. Notably, the Basal population exhibited a relative decrease in these pathways, suggesting that these cells may be less reliant on ErbB2 signaling for continued oncogenic proliferation or progression (Fig $5 A, B)$.

Instead, the Basal population showed enrichment of E2F and Myc signaling (Hallmarks), cell cycling (KEGG), G2/M checkpoint (Hallmarks), and p53 signaling (KEGG) pathways, consistent with proliferation, growth, and replicative stress (Fig 5A, B), as seen in bulk aggressive lesions (Fig 2D, E). Relative enrichment of spermatogenesis (Hallmarks) and oocyte meiosis (KEGG) pathways suggests inappropriate somatic upregulation of meiotic genes associated with disrupted mitosis (Bruggeman et al., 2018; Cappell et al., 2012; McFarlane and Wakeman, 2017; Zhang et al., 2020). As might be expected for rapidly cycling cells, the Basal population also exhibited enrichment of metabolic pathways (Fig $5 A, B)$, validated by increased levels of phosphorylated-S6 (Fig 4D). 
Consistent with our hypothesis that the LB population represents a mixed-lineage state, this population exhibited increased expression of genes associated with developmental and embryonic programs (Fig 5A, $B$ ), including Wnt-beta-catenin, Hedgehog, TGF-beta, and Notch, and Hippo signaling, as well epithelial-tomesenchymal transition (Hallmarks, KEGG).

Interestingly, we observed a modest enrichment of antigen processing and presentation (KEGG) and interferon-alpha signaling (Hallmarks) in the Luminal 1 population (Fig $5 A, B$ ). In contrast, the relative decrease of these pathways in the Basal population suggests that cells in the aggressive niche may be more resistant to immune surveillance, perhaps permitting their rapid expansion.

To identify whether Luminal 1 and Basal cells shift their function based on lesion aggressiveness, we analyzed these populations by lesion type and calculated the change in GSVA pathway enrichment scores in aggressive lesions relative to indolent lesions. We identified pathways for which both Luminal 1 and Basal populations were increased (e.g. Myc signaling) and decreased (e.g. apical surface) (Fig 5C, top panels) in aggressive lesions relative to indolent lesions. We also identified a set of pathways that were discordantly altered in aggressive populations. Specifically, oncogenic signaling and DNA stress pathways were increased in luminal but not basal populations in aggressive lesions relative to indolent lesions, whereas metabolic pathways were increased in basal but not luminal populations in aggressive lesions relative to indolent lesions (Fig 5C, bottom pane/s). Opposing pathway changes suggest that these tumor cell populations may play increasingly divergent functional roles within the lesion niche.

\section{The aggressive niche harbors an expanded and spatially-restricted neutrophil population}

To characterize the immune composition of indolent and aggressive lesions, we evaluated scRNA-seq data acquired from CD 45+ cells sorted by FACS from these two lesion types. We identified major immune cell types, including T cells (4 clusters), B cells, plasma cells, NK cells, neutrophils, macrophages, and dendritic cells (Fig 6A, B, D; Supp fig 5). The detection of a broad range of immune cell types in our model is consistent with previous findings in human breast cancer (Azizi et al., 2018; Chung et al., 2017; GattiMays et al., 2019; Gil Del Alcazar et al., 2020; Wagner et al., 2019). While these immune cell populations were identified in both lesion types, their relative frequencies were shifted (Fig 6C). To validate these findings, we performed immunostaining for several lymphoid and myeloid cells, including $T$ cells, $B$ cells, macrophages, and neutrophils (Fig 6E). In agreement with our scRNA-seq data, immunostaining showed decreased lymphoid cells ( $T$ cells, B cells) but increased myeloid cells (macrophages, neutrophils) in aggressive lesions (Fig 6C, E). These findings point to a lymphoid-to-myeloid shift consistent with the reported role of myeloid populations in mediating $T$ cell suppression. Indeed, although fewer infiltrating $\mathrm{CD} 4+$ and $\mathrm{CD} 8+\mathrm{T}$ cells per tumor area were observed in aggressive lesions relative to indolent lesions (Supp fig 6A-D), subset analysis of T cell clusters (T cell 1-4) revealed that immune-suppressed T cell populations were increased in relative frequency in aggressive lesions (Supp fig 6E-H). 
Validation by immunostaining for S100A8 (elevated in neutrophils and myeloid-derived suppressor cells (Ostrand-Rosenberg and Fenselau, 2018; Zhao et al., 2012)) showed that, among immune cells identified by staining, S100A8+ neutrophils exhibited the greatest dichotomy between indolent and aggressive niches (Fig 6F). Invasive niches exhibited a massive infiltration of S100A8+ neutrophils, while in situ lesions were almost completely devoid of these cells. Remarkably, the exclusion of S100A8+ neutrophils from in situ lesions was maintained even when these lesions were adjacent to their invasive counterparts, suggesting that the recruitment of these cells is tightly linked to the local aggressive niche.

Consistent with the active recruitment of neutrophils, we noted that one $T$ cell population ( $T$ cell 4 , potentially gamma-delta $T$ cells based on relatively increased expression of genes encoding gamma (Tcrg-C1) and delta (Trdc) T cell receptors, Fig 6D) displayed relatively higher expression of genes encoding IL-17 ligands, I/17a and I/17f (Fig 6D, Supp fig 7A, B). IL-17 is known to activate downstream signaling cascades ultimately leading to neutrophil recruitment. Genes encoding cognate receptors $/ / 17 \mathrm{ra}$ and $/ 117 \mathrm{rc}$ were relatively increased, respectively, in immune cells (particularly neutrophils and macrophages, Supp fig $7 C, D$ ) and Basal tumor cells (Supp fig $7 E, F$ ). Additionally, target genes downstream of IL-17 signaling (KEGG) was the most commonly increased pathway across multiple immune populations in aggressive lesions relative to indolent lesions (Fig 6G). These data identify a niche interaction in which IL-17 cytokines produced by one population may effect signaling pathways in potential niche collaborators. Altogether, our single-cell and histological findings suggest potential roles for IL-17 signaling and/or neutrophils in distinguishing indolent from aggressive lesions, and potentially driving or supporting aggressive lesion progression.

\section{Some indolent lesions bear tumor and immune features associated with aggressive lesion behavior}

Although we observed largely dichotomous features when comparing indolent with aggressive lesions, we did observe a subset of smaller-sized lesions that shared properties with aggressive lesions.

Specifically, some of these smaller lesions exhibited mixed-lineage cells and an expanded basal-like (cytokeratin 5) population, similar to that observed in advanced lesions (Supp fig 8A, B). Additionally, we observed a few rare smaller-sized lesions with accompanying neutrophils (Supp fig $8 C, D$ ). These observations suggest that the subset of indolent lesions exhibiting these features may be on their way to becoming aggressive lesions.

\section{Inhibition of granulocyte recruitment reduces aggressive niche formation and delays tumor progression}

Given the putative activation of the IL-17 pathway in our lesions in addition to its well-documented role in neutrophil recruitment, we tested the impact of IL-17-blockade on lesion progression. We treated mice with antibodies against IL-17A and its receptor IL-17RA (herein referred to as anti-IL-17), or isotype control, one week following intraductal injection with lentiviral caErbB2, a time when very early stage lesions are established (Fig $7 A)$. 
As previously observed, the majority of lesions across all animals (regardless of treatment) were small indolent lesions ( $93 \%$ less than $1 \mathrm{~mm}^{2}$ ), with only a minority rapidly progressing to a very large size (3.5\% at or greater than $10 \mathrm{~mm}^{2}$ ) (Fig $\left.7 B\right)$. Anti-IL-17 treated animals exhibited a decrease in the number of aggressive lesions $>1 \mathrm{~mm}^{2}$ (Fig $7 C, D$ ), associated with decreased circulating neutrophils (Fig 7E), suggesting that anti-IL-17 treatment inhibited lesion progression. A commensurate increase in small lesions (Fig 7C) suggests that IL-17 blockade did not prevent small lesions from forming but limited their subsequent progression. Finally, when compared with a population of animals identically treated with isotype control (pooled from multiple cohorts), animals treated with anti-IL-17 showed delayed latency to a palpable tumor (Fig 7F). These results are consistent with a pro-tumor role of IL-17 and possibly neutrophils in aggressive niches, as has been described for N2 neutrophils and/or granulocytic myeloid derived suppressor cells (G-MDSCs) (Furumaya et al., 2020; Ostrand-Rosenberg and Fenselau, 2018).

To validate the role of pro-tumor neutrophils/G-MDSCs in our model, we next measured the effect of treating lesion-bearing animals with SX-682, a small molecule inhibitor of CXCR1/2 reported to target GMDSCs (currently in phase I trials for melanoma, NCT03161431). After allowing lesions to establish for one week, we switched experimental mice to feed containing SX-682 or kept control mice on standard chow (Fig 7G). Again, the majority of lesions across all animals were small (97\%), with rapidly progressing lesions in minority $(<1 \%$, Fig $7 H)$. Compared to control animals, SX-682-treated animals harbored fewer lesions overall (Fig 7 ), as well as fewer aggressive lesions (Fig 7 l, $\mathrm{J}$ ), again associated with decreased circulating neutrophils (Fig $7 L$ ). SX-682-treated animals, when compared against a population of animals identically treated with standard chow, showed delayed latency to a palpable tumor (Fig 7K). Taken together, the decreased frequency of lesion advancement in animals treated with either anti-IL-17 or SX-682 suggest that IL-17 and neutrophils/G-MDSCs were capable of promoting aggressive niche formation and lesion progression.

\section{Discussion}

In this study, we utilized a mouse model of breast cancer that generated both indolent and aggressive lesions to gain insight on features that distinguish these lesion types and to identify potential determinants of lesion aggressiveness. We showed that indolent and aggressive lesions were comprised of distinct tumor-immune cell niches (Fig 8). Importantly, these distinct niches existed within the same mammary gland, strongly supporting the idea that niche features can be locally determined by spatiallyrestricted collaborative cues that modulate or override systemic conditions and, in this model, a common tumor-initiating event (expression of caErbb2).

We demonstrated that lesions harbor a heterogeneous population of tumor cells that shift in frequency in aggressive lesions relative to indolent lesions. Further, we inferred that luminal-like and basal-like populations within lesions adopt divergent biological roles that become more extreme during disease progression, gaining insight into the functional specialization that accompanies increased cellular, spatial, and resource heterogeneity within the tumor niche (Kotler and Brown, 2020). Such insight has been challenging to gain from genomic and transcriptomic analyses that have identified high 
concordance detected between invasive and non-invasive lesions, but resonate with observations of ecological niche partitioning and division of labor (Rosenthal et al., 2018).

We found that aggressive lesions harbored mixed-lineage tumor cells. The emergence of these cells are consistent with similar mixed-lineage populations detected in recent single-cell studies of normal and transformed mammary tissues (Bach et al., 2017; Chung et al., 2019; Giraddi et al., 2018; Nguyen et al., 2018; Pal et al., 2017; Sun et al., 2017; Thong et al., 2020; Yeo et al., 2020), and suggest that aggressive progression in this model is accompanied by increased tumor cell plasticity. In particular, our observations agree with previous findings that luminal-like cells acquire basal-like features following oncogenic or homeostatic disruption (Hein et al., 2016; Lim et al., 2009; Meyer et al., 2011; Molyneux et al., 2010; Van Keymeulen et al., 2015; Wahl and Spike, 2017; Wuidart et al., 2018). Conversely, we cannot rule out the possibility that the LB population arises from basal-like cells, consistent with basalcompartment cells giving rise to luminal cells in proposed hierarches of normal mammary gland development (Fu et al., 2020; Regan and Smalley, 2020), and with studies showing that adult committed basal cells can be reprogrammed to acquire more luminal-like states (Koren et al., 2015; Lilja et al., 2018; Van Keymeulen et al., 2015).

Additionally, LB cells may exhibit progenitor-like properties, as suggested by findings that epithelial-tomesenchymal programs can generate cells with stem-like properties (Mani et al., 2008). Such hybrid cells have been shown to promote aggressive behavior, by enhancing tumor initiation, invasion, metastasis, intratumoral heterogeneity, and therapy-resistance (Brooks et al., 2015; Jolly et al., 2019; Kong et al., 2020; Kroger et al., 2019; Risom et al., 2018). These studies and our work highlight the need to define and functionalize various cell states and lineages partaking in breast tumor progression, including those identified in our study. To this end, future work aims to more fully characterize these hybrid mixed-lineage cells in our model and measure their biological potential.

Despite vast tumor cell-intrinsic differences between indolent and aggressive lesions, we found that cells derived from these distinct lesions were functionally indistinguishable from one another when placed in matched microenvironments. These results support a model of conditional lesion aggressiveness in which crosstalk between tumor cells and their local niche play a more critical role in driving aggressive behavior than do major cell-intrinsic transcriptional and phenotypic differences between indolent and aggressive tumor cells (Bissell and Hines, 2011). Our observations are in agreement with previous reports that human in situ lesions may harbor occult cells capable of aggressive behavior and invasive growth, and that genetically-similar lesions may nonetheless differ in local aggressiveness (in situ versus invasive) (Buerger et al., 1999; Casasent et al., 2018; Cowell et al., 2013; Espina et al., 2010; Ma et al., 2003; Vincent-Salomon et al., 2008). Thus, this study underscores the ongoing need to understand the role of the tumor niche in driving lesion behavior even in early stages of breast cancer progression, and to determine how re-shaping the local niche might prevent or control aggressive tumor behavior.

To this end, we described a shift in immune composition between indolent and aggressive lesions, in agreement with previous reports (Campbell et al., 2017; Gil Del Alcazar et al., 2017). Notably, we observed 
that aggressive lesions exhibited a lymphoid-to-myeloid switch consistent with findings that tumors recruit myeloid cells that can suppress anti-tumor lymphocytes (Apolloni et al., 2000; Bronte et al., 1998; Gabrilovich et al., 2001; Katzenelenbogen et al., 2020; Molgora et al., 2020; Veglia et al., 2018), and that tumor aggressiveness, including the transition from in situ to invasive disease, is modulated by the ability of a lesion niche to dampen anti-tumor immunity (Gil Del Alcazar et al., 2020; Gil Del Alcazar et al., 2017).

In particular, we identified that S100A8+ neutrophils specifically infiltrated aggressive niches, which also displayed increased IL-17 signaling. Tumor-associated neutrophils, or TANs, have been reported to play dual roles in the tumor microenvironment, including in breast cancer (Fridlender et al., 2009; Furumaya et al., 2020; Jaillon et al., 2020; Mishalian et al., 2013). The functional role of TANs in DCIS, which have been shown to increase compared to normal tissue, is not known, but may be similarly double-sided (Gil Del Alcazar et al., 2017; Nelson et al., 2018). Additionally, IL-17 signaling may impact tumor cells, for example by regulating tumor cell plasticity, as has been shown in pancreatic lesions (Zhang et al., 2018). Recent studies have found that IL-17 and TANs can promote the progression of tumors derived from transplanted metastatic or chemoresistant cells (Benevides et al., 2015; Wu et al., 2020). In agreement with these findings, in a proof-of-principle study using our in vivo model of spontaneous tumor initiation, we determined that IL-17 and/or TANs played a pro-tumor role even at early stages of aggressive progression, when lesions become locally invasive. Notably, TANs were very tightly associated with the establishment of the aggressive niche such that we could not dissociate these two niche features even with blockade of neutrophil recruitment. Altogether, our work and others suggest that IL-17 signaling and neutrophil recruitment promote tumor aggressiveness as early as the onset of local invasion, through to metastasis and development of chemoresistance, indicating a large therapeutic window during which targeting IL-17/TANs may yield clinical benefit. Thus, our work warrants further mechanistic investigation of IL-17 signaling and TANs to identify or therapeutically target DCIS lesions that may be capable of aggressive behavior, including the use of SX-682 in patients at risk for aggressive progression. Importantly, since inhibiting neutrophil recruitment reduced but did not eliminate aggressive lesions, it will be essential to account for the likely contributions of additional niche collaborators, including both cellular and acellular elements such as altered matrix structure and composition (McAllister and Weinberg, 2014; Quail and Joyce, 2013; Welte et al., 2016; Zhang et al., 2012; Zhang et al., 2018).

Finally, although our findings do not rule out the possibility that indolent and aggressive lesions follow independent natural histories, our observations suggest a putative biological continuum between some indolent and aggressive lesions based on the identification of small lesions bearing aggressive niche features. Importantly, these features may also herald aggressiveness in human lesions that might be otherwise considered indolent. Ongoing work includes profiling human breast lesions in various stages of progression to determine whether and at which point such features arise, to explore their potential clinical utility as predictive markers to strengthen current risk assessment approaches (Hanna et al., 2019), and to identify whether the populations they mark could serve as therapeutic targets to prevent or delay disease progression. Because our model histologically resembles aggressive metaplastic breast cancer that in rare cases also expresses HER2 (Weigelt et al., 2014), similar studies are required in additional models to determine the relationship between indolent and aggressive lesion niche collaborators across a 
broader spectrum of breast cancer subtypes, and to identify key potential niche targets for treatment stratification and therapeutic intervention.

\section{Declarations}

\section{Acknowledgements}

We thank members of the $\mathrm{H}$. Piwnica-Worms' lab, including former members Emily Powell, Abena B. Redwood, Gloria V. Echeverria, Kristina M. Stemler, and Marimar de la Cruz Bonilla for helpful discussions throughout the course of this study. We thank Yi Li for mentorship and providing the lentiviral vector plasmid; Jeffrey M. Rosen for advice throughout the project; Anne Meyn for providing perspective as a patients advocate; Elizabeth A. Mittendorf and Han Liang for advice during project conception; Jared Burks, Duncan Mak, and Angelique Lin for training and support on multiparametric imaging and antibody conjugations; Yun Wu for pathology input; Zhongqi Ge for preliminary bioinformatics support; and Jie Zhang for assistance in processing animal tissues. BioRender was used to generate summary figures. This work was supported in part by the Stand Up To Cancer Laura Ziskin Prize (to H.P.-W.), the Cancer Prevention and Research Institute of Texas RP200120 (to H.P.-W.), the National Institutes of Health (NIH) T32CA186892 (to V.C.S.) and by the Department of Defense W81XWH-17-1-0077 (to V.C.S.). Opinions, interpretations, conclusions, and recommendations are those of the authors and are not necessarily endorsed by the Department of Defense. The MD Anderson Flow Cytometry and Cellular Imaging Core Facility, Research Histology Core Laboratory, and Advanced Technology Genomics Core are supported by NIH P30CA16672. The University of Michigan Medical School Biomedical Research Core Facilities are supported by NIH P30CA046592 and P30DK034933.

\section{Author contributions}

Conceptualization: V.C.S., H.P.-W.; Formal analysis: V.C.S., M.X., A.L.R., Y.Q.; Investigation: V.C.S., A.L.R., X.Zhou, X.Zhang, S.J.J., J.S.; Resources: F.M., J.A.Z, D.Y.M; Writing - Original draft: V.C.S.; Writing Reviewing and editing: V.C.S., A.L.R., H.P.-W.; Supervision: H.P.-W., F.M.; Funding acquisition: H.P.-W., V.C.S.

\section{Declaration of Interests}

The authors declare no competing interests.

\section{Methods}

\section{Animals}

Animal work described was performed in accordance with recommendations in the Guide for Care and Use of Laboratory Animals from the National Institutes of Health $(\mathrm{NIH})$ Institutional Animal Care and Use Committee (IACUC) and the American Association for Accreditation of Laboratory Animal Care (AAALAC). Studies were approved by the University of Texas MD Anderson Cancer Center IACUC and, where 
applicable, the U.S. Army Medical Research and Development Command (USAMRDC) Animal Care and Use Review Office (ACURO). Animals were obtained from Jackson Laboratories (FVB/NJ, 001800) for direct use in studies as well as establishment of in-house colonies, and all housing and work carried out in an AAALAC International-accredited and Public Health Service (PHS) Animal Welfare-assured facility. All animals were euthanized in accordance with $\mathrm{NIH}$ and AAALAC guidelines.

\section{Lentivirus production and titering}

Constitutively activated ErbB2 (ca-ErbB2) was delivered using a lentiviral vector (FUCGW) as previously described (Bu et al., 2009). Virus was produced in-house as previously described (Bu et al., 2009; Du et al., 2006) or prepared by the University of Michigan Medical School Biomedical Research Core Facilities. For in-house preparation, lentiviral expression and packaging plasmids ( $p M D L g / p R R E, p R S V-R e v, V S V g$ ) were transfected with TransIT ${ }^{\circledR}$-LT1 (Mirus) or FuGENE® 6 (Promega) into HEK293T cells. Virus-containing media was filtered through $0.45 \mu \mathrm{m}$ membrane then subjected to centrifugation at $70,000 \mathrm{~g}$ for 3 hours to concentrate. Concentrated virus was titered by limiting dilution transduction of $293 \mathrm{~T}$ cells, followed by enumeration of percent fluorescent cells. Aliquots of concentrated virus were stored at $-80^{\circ} \mathrm{C}$ until use. Work described was approved by the MD Anderson Cancer Center Institutional Biosafety Committee.

\section{Intraductal injection of virus/cells}

Adult female virgin FVB mice were anesthetized using isoflurane (2-4\%) by way of inhalation. Following confirmation of anesthesia by gentle toe pinch, a small tip $(<3 \mathrm{~mm})$ of the \#4 nipple was cut with microdissecting surgical scissors to expose the nipple duct. Up to $1 \times 10^{6}$ viral particles or 200,000 cells ( 10 ul volume) were injected into the nipple duct using 22- to 34-gauge blunt needles fitted to a Hamilton syringe. Mice were palpated for tumors up to three times weekly until study endpoint.

\section{Tissue processing (embedding)}

Upon collection of mammary glands, tissues were incubated in $10 \%$ formalin at $4^{\circ} \mathrm{C} \sim 48-72$ hours until fixation was achieved, then embedded in paraffin. Formalin-fixed paraffin-embedded (FFPE) tissues were sectioned to 3-5 $\mu \mathrm{m}$ in-house, or by the MD Anderson Research Histology Core Laboratory (RHCL), or the Center for Radiation Oncology Research (CROR) Histology Core.

\section{Tissue staining (H\&E and Immunostaining)}

Hematoxylin and eosin (H\&E) staining was performed by the RHCL or CROR Histology Core. H\&E stained slides were scanned at MD Anderson RHCL using the Aperio AT2 slide scanner (Leica Biosystems), and lesions digitally quantified using Aperio ImageScope (Leica Biosystems) or ImageJ (Schneider et al., 2012). To perform immunostaining, FFPE tissue slides were baked at $65^{\circ} \mathrm{C}$ for an hour, and then dewaxed and rehydrated by graded washes in xylene to ethanol. Heat-mediated epitope retrieval was performed by incubating slides in Reveal Decloaker or Nuclear Decloaker solutions (Biocare Medical), heated to $97^{\circ} \mathrm{C}$ for 15 minutes using an EZ-Retriever microwave (BioGenex). Following phosphate-buffered saline (PBS) 
wash, endogenous peroxidase activity was quenched by incubation of tissues in Dual Endogenous Enzyme Block (Dako) for 10 minutes at room temperature. Tissues were blocked using Protein Block (Dako) and/or normal serum (Vector ImmPRESS). Slides were incubated with primary antibodies overnight at $4^{\circ} \mathrm{C}$, washed in PBS, then incubated with secondary antibodies. For immunofluorescence (IF), slides were mounted using ProLong ${ }^{\text {TM }}$ Gold Antifade Mountant with DAPI (Thermo Fisher). For immunohistochemistry (IHC), slides were incubated with horse radish peroxidase substrate (HRP, Vector ImmPACT) to develop stain, counterstained with hematoxylin QS (Vector), dehydrated through graded ethanol-to-xylene washes, and then mounted using permanent mounting medium (VectraMount). For multiplex IF, slides were prepared using the Opal 7-color Manual IHC Kit per manufacturer's instructions (Akoya Biosciences). Briefly, following incubation with HRP, slides were stained with tyramide-bound fluorophore, and then treated with additional rounds of HIER, re-staining until finally counterstained with DAPI and mounted with ProLong ${ }^{\text {TM }}$ Gold Antifade Mountant with DAPI (Thermo Fisher). Slides were imaged using the Vectra ${ }^{\circledR} 3$ (Akoya Biosciences) or Vectra ${ }^{\circledR}$ Polaris ${ }^{\mathrm{TM}}$ Quantitative Pathology Imaging System (Akoya Biosciences) housed at the MD Anderson Flow Cytometry and Cellular Imaging Core Facility (FCCICF). Slides stained for imaging mass cytometry (Giesen et al., 2014) were prepared as above for IHC up to incubation with a freshly-prepared cocktail of metal-conjugated primary antibodies, after which slides were washed in PBS, counterstained with 0.625 uM iridium in $1.6 \%$ paraformaldehyde for 30 minutes at room temperature and then air dried. Tissue sections stained with metal-conjugated antibodies were scanned using the Hyperion Imaging System (Fluidigm) housed at the FCCICF, and image data visualized and processed using MCD Viewer (Fluidigm). Images were processed and compiled using Adobe Photoshop and Adobe Illustrator.

The following antibodies were used for immunostaining with IHC, IF, and multiplex IF: CD19 (Thermo Fisher 14-0194-82); CD3 (Abcam ab5690); CD45 (e-Biosciences 14-0451-85); Col IV (Abcam ab6586); Ecadherin (Cell Signaling Technology 3195); ER-alpha (Millipore 06-935); F4/80 (Thermo MA1-91124); HA (BioLegend 901502); Ki67 (Abcam ab15580); MPO (Abcam ab208670); S100A8 (R\&D Systems MAB3059). The following antibodies were used for imaging mass cytometry: CD163 (Abcam ab213612), conjugated to $168 \mathrm{Er}$; CK5 (Abcam ab214586), 145 Nd; CK8 (Abcam ab217173), 154 Sm; Col-IV (Abcam ab6586), 155 Gd; pS6 (CST 4858), 167 Er; S100A8 (R\&D MAB3059), 115 In; Vimentin (Abcam 193555), $151 \mathrm{Eu}$.

\section{Antibody conjugation}

Antibody conjugations were performed at the FCCICF using Maxpar Antibody Labeling reagents (Fludigm) as previously described (Wei et al., 2017). Briefly, commercially obtained carrier-free antibodies were prepared for conjugation through partial reduction of antibody sulfide bonds (TCEP, Sigma) then conjugated to metal-loaded polymer (Fluidigm). Antibody-metal conjugates were washed, quantified by Nanodrop, recovered to $0.5 \mathrm{mg} / \mathrm{ml}$ in antibody stabilizer (Candor Biosciences), and stored at $4^{\circ} \mathrm{C}$.

\section{Tissue processing (digestion)}


Resected mammary glands bearing caErbB2-GFP lesions were visualized under a fluorescence stereoscope, and regions harboring aggressive ( $>2 \mathrm{~mm}$, comprised of invasive lesions) and indolent ( $<2 \mathrm{~mm}$, enriched for in situ lesions) lesions were dissected away from each other. Tissues were mechanically minced, and then digested to organoids in epithelial cell media [advanced DMEM/F12 (Gibco) with $5 \%$ fetal bovine serum (Hyclone), and $1 \%$ antibiotic-antimycotic (Corning)], supplemented with $3 \mathrm{mg} / \mathrm{ml}$ collagenase (Roche), $0.6 \mathrm{mg} / \mathrm{ml}$ hyaluronidase (Sigma), 1.3\% bovine serum albumin (BSA) (Sigma). Tissue digests were agitated by angled rotation for $2-5$ hours at $37^{\circ} \mathrm{C}$, pelleted by centrifugation at $450 \mathrm{~g}$, and then resuspended in red cell lysis buffer (Sigma). Following wash with epithelial cell media and PBS, pelleted organoids were incubated in $0.25 \%$ Trypsin-EDTA (Corning) at room temperature for 5 minutes. Cells were again washed, pelleted, and resuspended in epithelial cell media supplemented with up to $10 \mathrm{U}$ dispase (Stem Cell) and 5ug DNAase I (Stem Cell), filtered serially through $100 \mu \mathrm{m}$ and $70 \mu \mathrm{m}$ cell strainers (Falcon), and enumerated by trypan blue staining.

\section{Preparation of isolated cells for injection}

Following digestion of tissues down to single cells, samples were stored at $4^{\circ} \mathrm{C}$ overnight in Hypothermosol (BioLife Solutions). The following day, tumor cells were washed and resuspended in PBS with $0.5 \%$ EDTA, and then isolated by fluorescence-activated cell sorting (FACS) for GFP. Sorted cells were washed with HBSS supplemented with 5\% FBS, $10 \mu \mathrm{M}$ ROCK inhibitor (Sigma), and $500 \mu \mathrm{m} \mathrm{N}$ acetylcysteine amide (NAC) (Sigma). For transplantation studies, cells were resuspended in HBSS only and kept on ice until same-day injection.

\section{Bulk RNA sequencing}

For bulk transcriptomic profiling, digested tumor cells were isolated by FACS as above, homogenized using a Bullet Blender (Next Advance) and RNA extracted (Qiagen RNeasy Kit). RNA was treated with DNase (Turbo DNA-Free Kit), and then cleaned and concentrated (Zymo RNA Cleanup Kit), per manufacturers' instructions. RNA library preparation (NEBNext® Ultra ${ }^{\text {TM }}$ RNA Library Prep Kit for Illumina, NEBNext ${ }^{\circledR}$ Poly(A) mRNA Magnetic Isolation Module) and sequencing (Illumina HiSeqX Ten, 150 nucleotide paired-end reads) were performed by Admera Health (South Plainfield, NJ). STAR (version 2.6.0b) (Dobin et al., 2013) was used to align reads to the GRCm38.p6 reference genome. Samtools (version 1.8) (Li et al., 2009) was used to sort, convert between formats, and calculate mapping statistics. FastQC (version 0.11.5) (Andrews, 2010) was used to check for qualities of the FASTQ reads. Gene annotation was carried out using the GENCODE M19 (Ensembl 94) annotation, which was downloaded from the GENCODE project(Harrow et al., 2012). Aligned reads were summarized at the gene level by STAR (version 2.6.0b) (Dobin et al., 2013). R (3.6.0) and Bioconductor package DESeq2 (Love et al., 2014) was used to identify differentially expressed genes. Read count was first pre-filtered to remove extremely low expressed genes. DESeq2 then carried out read count filtering, normalization, dispersion estimation, and identification of differential expression. DESeq2 modeled the counts using a negative binomial distribution, followed by the Wald test. The final p-value was adjusted using the Benjamini \& Hochberg method. Heatmaps of unsupervised hierarchical clustering of differentially expressed genes in all 
samples were plotted. The genes were median-centered for contrast. The clustering was done using Pearson distance and Ward linkage. The Molecular Signatures Database (MSigDB, https://www.gseamsigdb.org/gsea/msigdb/index.jsp) v7.0 hallmark gene sets and curated gene sets (c2) were used in Gene Set Enrichment Analysis (GSEA).

\section{Single-cell RNA sequencing}

For single-cell transcriptomic profiling, tumor and immune cells were isolated by FACS for GFP and CD 45 . Samples were transferred in media suspension to the MD Anderson Advanced Technology Genomics Core (ATGC, CA016672), where sample concentration and viability were evaluated using automated cell counting (Countess II FL Auto counter), prior to 3' capture and library preparation (5000 cells/sample, Chromium Single Cell 3' v2) using the Chromium Controller (10X Genomics) and sequencing (Illumina NextSeq500). Raw FASTQ reads were mapped to mouse mm10 genome by Cell Ranger v3.0.2 to generate the cell-gene count matrix (Zheng et al., 2017). The matrix file was analyzed by R package Seurat v3.1.0 (Stuart et al., 2019). Specifically, cells with less than 500 UMI count or more than $10 \%$ mitochondrial gene UMI count were treated as low-quality cells and excluded from further analysis. Cell cycle scores ( $\mathrm{S}$ and G2M) were calculated using the gene lists provided by Seurat. The count matrix was then log normalized and multiplied by the scale factor 10,000. Variance-stabilizing transformation (VST) method was used to choose the top 2,000 variable genes. The package DoubleFinder was used to exclude cells likely to be doublets (McGinnis et al., 2019). A doublet percentage of $5 \%$ was used for the estimation. The cells from the same FACS strategy (GFP or CD45) were integrated with Canonical Correlation Analysis (CCA) and dimension of 30 . The integrated data matrices were further scaled and centered, and the effects of UMI counts, mitochondrial RNA proportion, cell cycle scores were also regressed out during this step. The data were further processed and visualized by Principle Component Analysis (PCA) and Uniform Manifold Approximation and Projection (UMAP). Smart local moving (SLM) algorithm was used for cluster identification. Differential expression (DE) analysis between groups in each cluster was performed using FindMarkers function. GSVA scores were calculated by R package GSVA v1.36.2 (Hanzelmann et al., 2013). The Hallmarks and KEGG gene lists were downloaded from MSigDB v7.0. Pseudotime analysis was performed by Monocle v2 (Qiu et al., 2017a). T cell exhaustion scores were calculated by Seurat AddModuleScore function and T cell exhaustion gene lists from a previous study (Tirosh et al., 2016).

\section{In vivo antibody/diet studies}

Lentiviral particles were injected intraductally as described above, and lesions were allowed to establish for one week, after which treatment commenced. For IL-17 blockade studies, 200ug each of anti-IL-17 and anti-IL-17R antibodies (Amgen) (or polyclonal IgG control, BioXcell) in PBS were administered intraperitoneally twice per week until study endpoint. For SX-682 studies, experimental animals were placed on medicated diet (Syntrix Pharmaceuticals) formulated with 0.756 grams of SX-682 per kilogram of feed until study endpoint. Food consumption was measured twice weekly to calculate approximate mean daily dose $(\sim 80 \mathrm{mg} / \mathrm{kg})$. For both studies, mammary glands were palpated twice weekly for tumors and animals were monitored for potential adverse effects including body weight loss. When the most 
rapidly growing tumors reached maximum allowable burden, mammary tissues from all animals were collected and then formalin-fixed and paraffin-embedded (FFPE) as described above.

\section{Data and software availability.}

Sequencing data can be accessed under GSE162005 and GSE161983. (Data will be publically released upon acceptance.)

\section{Contact for Reagent and Resource Sharing}

Further information and requests for resources and reagents should be directed to and will be fulfilled by the Lead Contact, Helen Piwnica-Worms (hpiwnica-worms@mdanderson.org).

\section{References}

Allen, M. D., Thomas, G. J., Clark, S., Dawoud, M. M., Vallath, S., Payne, S. J., Gomm, J. J., Dreger, S. A., Dickinson, S., Edwards, D. R., et al. (2014). Altered microenvironment promotes progression of preinvasive breast cancer: myoepithelial expression of alphavbeta6 integrin in DCIS identifies high-risk patients and predicts recurrence. Clin Cancer Res 20,344-357.

Andrews, S. (2010). FastQC: A Quality Control Tool for High Throughput Sequence Data [Online]. Available: http://www.bioinformatics.bbsrc.ac.uk/projects/fastqc [Accessed 2020].

Apolloni, E., Bronte, V., Mazzoni, A., Serafini, P., Cabrelle, A., Segal, D. M., Young, H. A., and Zanovello, P. (2000). Immortalized myeloid suppressor cells trigger apoptosis in antigen-activated T lymphocytes. J Immunol 165, 6723-6730.

Azizi, E., Carr, A. J., Plitas, G., Cornish, A. E., Konopacki, C., Prabhakaran, S., Nainys, J., Wu, K., Kiseliovas, V., Setty, M., et al. (2018). Single-Cell Map of Diverse Immune Phenotypes in the Breast Tumor Microenvironment. Cell 174, 1293-1308 e1236.

Bach, K., Pensa, S., Grzelak, M., Hadfield, J., Adams, D. J., Marioni, J. C., and Khaled, W. T. (2017). Differentiation dynamics of mammary epithelial cells revealed by single-cell RNA sequencing. Nat Commun 8, 2128.

Benevides, L., da Fonseca, D. M., Donate, P. B., Tiezzi, D. G., De Carvalho, D. D., de Andrade, J. M., Martins, G. A., and Silva, J. S. (2015). IL17 Promotes Mammary Tumor Progression by Changing the Behavior of Tumor Cells and Eliciting Tumorigenic Neutrophils Recruitment. Cancer Res 75, 3788-3799.

Bissell, M. J., and Hines, W. C. (2011). Why don't we get more cancer? A proposed role of the microenvironment in restraining cancer progression. Nat Med 17, 320-329.

Bronte, V., Wang, M., Overwijk, W. W., Surman, D. R., Pericle, F., Rosenberg, S. A., and Restifo, N. P. (1998). Apoptotic death of CD8+ T lymphocytes after immunization: induction of a suppressive population of 
Brooks, M. D., Burness, M. L., and Wicha, M. S. (2015). Therapeutic Implications of Cellular Heterogeneity and Plasticity in Breast Cancer. Cell Stem Cell 17, 260-271.

Bruggeman, J. W., Koster, J., Lodder, P., Repping, S., and Hamer, G. (2018). Massive expression of germ cell-specific genes is a hallmark of cancer and a potential target for novel treatment development. Oncogene 37, 5694-5700.

Bu, W., Xin, L., Toneff, M., Li, L., and Li, Y. (2009). Lentivirus vectors for stably introducing genes into mammary epithelial cells in vivo. J Mammary Gland Biol Neoplasia 14, 401-404.

Buerger, H., Otterbach, F., Simon, R., Poremba, C., Diallo, R., Decker, T., Riethdorf, L., Brinkschmidt, C., Dockhorn-Dworniczak, B., and Boecker, W. (1999). Comparative genomic hybridization of ductal carcinoma in situ of the breast-evidence of multiple genetic pathways. J Pathol 187, 396-402.

Campbell, M. J., Baehner, F., O'Meara, T., Ojukwu, E., Han, B., Mukhtar, R., Tandon, V., Endicott, M., Zhu, Z., Wong, J., et al. (2017). Characterizing the immune microenvironment in high-risk ductal carcinoma in situ of the breast. Breast Cancer Res Treat 161, 17-28.

Cappell, K. M., Sinnott, R., Taus, P., Maxfield, K., Scarbrough, M., and Whitehurst, A. W. (2012). Multiple cancer testis antigens function to support tumor cell mitotic fidelity. Mol Cell Biol 32, 4131-4140.

Casasent, A. K., Schalck, A., Gao, R., Sei, E., Long, A., Pangburn, W., Casasent, T., Meric-Bernstam, F., Edgerton, M. E., and Navin, N. E. (2018). Multiclonal Invasion in Breast Tumors Identified by Topographic Single Cell Sequencing. Cell 172, 205-217 e212.

Chung, C. Y., Ma, Z., Dravis, C., Preissl, S., Poirion, O., Luna, G., Hou, X., Giraddi, R. R., Ren, B., and Wahl, G. M. (2019). Single-Cell Chromatin Analysis of Mammary Gland Development Reveals Cell-State Transcriptional Regulators and Lineage Relationships. Cell Rep 29, 495-510 e496.

Chung, W., Eum, H. H., Lee, H. O., Lee, K. M., Lee, H. B., Kim, K. T., Ryu, H. S., Kim, S., Lee, J. E., Park, Y. H., et al. (2017). Single-cell RNA-seq enables comprehensive tumour and immune cell profiling in primary breast cancer. Nat Commun 8, 15081.

Cowell, C. F., Weigelt, B., Sakr, R. A., Ng, C. K., Hicks, J., King, T. A., and Reis-Filho, J. S. (2013). Progression from ductal carcinoma in situ to invasive breast cancer: revisited. Mol Oncol 7, 859-869.

DeSantis, C. E., Ma, J., Gaudet, M. M., Newman, L. A., Miller, K. D., Goding Sauer, A., Jemal, A., and Siegel, R. L. (2019). Breast cancer statistics, 2019. CA Cancer J Clin 69, 438-451.

Dobin, A., Davis, C. A., Schlesinger, F., Drenkow, J., Zaleski, C., Jha, S., Batut, P., Chaisson, M., and Gingeras, T. R. (2013). STAR: ultrafast universal RNA-seq aligner. Bioinformatics 29, 15-21. 
Du, Z., Podsypanina, K., Huang, S., McGrath, A., Toneff, M. J., Bogoslovskaia, E., Zhang, X., Moraes, R. C., Fluck, M., Allred, D. C., et al. (2006). Introduction of oncogenes into mammary glands in vivo with an avian retroviral vector initiates and promotes carcinogenesis in mouse models. Proc Natl Acad Sci U S A 103, 17396-17401.

Elshof, L. E., Tryfonidis, K., Slaets, L., van Leeuwen-Stok, A. E., Skinner, V. P., Dif, N., Pijnappel, R. M., Bijker, N., Rutgers, E. J., and Wesseling, J. (2015). Feasibility of a prospective, randomised, open-label, international multicentre, phase III, non-inferiority trial to assess the safety of active surveillance for low risk ductal carcinoma in situ - The LORD study. Eur J Cancer 51, 1497-1510.

Emery, L. A., Tripathi, A., King, C., Kavanah, M., Mendez, J., Stone, M. D., de las Morenas, A., Sebastiani, P., and Rosenberg, C. L. (2009). Early dysregulation of cell adhesion and extracellular matrix pathways in breast cancer progression. Am J Pathol 175, 1292-1302.

Espina, V., Mariani, B. D., Gallagher, R. I., Tran, K., Banks, S., Wiedemann, J., Huryk, H., Mueller, C., Adamo, L., Deng, J. H., et al. (2010). Malignant Precursor Cells Pre-Exist in Human Breast DCIS and Require Autophagy for Survival. PLoS One 5, e10240.

Francis, A., Thomas, J., Fallowfield, L., Wallis, M., Bartlett, J. M., Brookes, C., Roberts, T., Pirrie, S., Gaunt, C., Young, J., et al. (2015). Addressing overtreatment of screen detected DCIS; the LORIS trial. Eur J Cancer $51,2296-2303$.

Fridlender, Z. G., Sun, J., Kim, S., Kapoor, V., Cheng, G., Ling, L., Worthen, G. S., and Albelda, S. M. (2009). Polarization of tumor-associated neutrophil phenotype by TGF-beta: "N1" versus "N2" TAN. Cancer Cell 16, 183-194.

Fu, N. Y., Nolan, E., Lindeman, G. J., and Visvader, J. E. (2020). Stem Cells and the Differentiation Hierarchy in Mammary Gland Development. Physiol Rev 100, 489-523.

Furumaya, C., Martinez-Sanz, P., Bouti, P., Kuijpers, T. W., and Matlung, H. L. (2020). Plasticity in Pro- and Anti-tumor Activity of Neutrophils: Shifting the Balance. Front Immunol 11, 2100.

Gabrilovich, D. I., Velders, M. P., Sotomayor, E. M., and Kast, W. M. (2001). Mechanism of immune dysfunction in cancer mediated by immature Gr-1+ myeloid cells. J Immunol 166, 5398-5406.

Gatti-Mays, M. E., Balko, J. M., Gameiro, S. R., Bear, H. D., Prabhakaran, S., Fukui, J., Disis, M. L., Nanda, R., Gulley, J. L., Kalinsky, K., et al. (2019). If we build it they will come: targeting the immune response to breast cancer. NPJ Breast Cancer 5, 37.

Gerdes, M. J., Gokmen-Polar, Y., Sui, Y., Pang, A. S., LaPlante, N., Harris, A. L., Tan, P. H., Ginty, F., and Badve, S. S. (2018). Single-cell heterogeneity in ductal carcinoma in situ of breast. Mod Pathol 31, 406417. 
Giesen, C., Wang, H. A., Schapiro, D., Zivanovic, N., Jacobs, A., Hattendorf, B., Schuffler, P. J., Grolimund, D., Buhmann, J. M., Brandt, S., et al. (2014). Highly multiplexed imaging of tumor tissues with subcellular resolution by mass cytometry. Nat Methods 11, 417-422.

Gil Del Alcazar, C. R., Aleckovic, M., and Polyak, K. (2020). Immune Escape during Breast Tumor Progression. Cancer Immunol Res 8, 422-427.

Gil Del Alcazar, C. R., Huh, S. J., Ekram, M. B., Trinh, A., Liu, L. L., Beca, F., Zi, X., Kwak, M., Bergholtz, H., Su, Y., et al. (2017). Immune Escape in Breast Cancer During In Situ to Invasive Carcinoma Transition. Cancer Discov 7, 1098-1115.

Giraddi, R. R., Chung, C. Y., Heinz, R. E., Balcioglu, O., Novotny, M., Trejo, C. L., Dravis, C., Hagos, B. M., Mehrabad, E. M., Rodewald, L. W., et al. (2018). Single-Cell Transcriptomes Distinguish Stem Cell State Changes and Lineage Specification Programs in Early Mammary Gland Development. Cell Rep 24, 16531666 e1657.

Goldhirsch, A., Wood, W. C., Coates, A. S., Gelber, R. D., Thurlimann, B., Senn, H. J., and Panel, m. (2011). Strategies for subtypes-dealing with the diversity of breast cancer: highlights of the St. Gallen International Expert Consensus on the Primary Therapy of Early Breast Cancer 2011. Ann Oncol 22, 17361747.

Han, M. S., and Khan, S. A. (2018). Clinical Trials for Ductal Carcinoma In Situ of the Breast. J Mammary Gland Biol Neoplasia 23, 293-301.

Hanahan, D., and Weinberg, R. A. (2011). Hallmarks of Cancer: The Next Generation. Cell 144, 646-674.

Hanna, W. M., Parra-Herran, C., Lu, F. I., Slodkowska, E., Rakovitch, E., and Nofech-Mozes, S. (2019). Ductal carcinoma in situ of the breast: an update for the pathologist in the era of individualized risk assessment and tailored therapies. Mod Pathol 32, 896-915.

Hanzelmann, S., Castelo, R., and Guinney, J. (2013). GSVA: gene set variation analysis for microarray and RNA-seq data. BMC Bioinformatics 14, 7.

Harrow, J., Frankish, A., Gonzalez, J. M., Tapanari, E., Diekhans, M., Kokocinski, F., Aken, B. L., Barrell, D., Zadissa, A., Searle, S., et al. (2012). GENCODE: the reference human genome annotation for The ENCODE Project. Genome Res 22, 1760-1774.

Hein, S. M., Haricharan, S., Johnston, A. N., Toneff, M. J., Reddy, J. P., Dong, J., Bu, W., and Li, Y. (2016). Luminal epithelial cells within the mammary gland can produce basal cells upon oncogenic stress. Oncogene 35, 1461-1467.

Heselmeyer-Haddad, K., Berroa Garcia, L. Y., Bradley, A., Ortiz-Melendez, C., Lee, W. J., Christensen, R., Prindiville, S. A., Calzone, K. A., Soballe, P. W., Hu, Y., et al. (2012). Single-cell genetic analysis of ductal 
carcinoma in situ and invasive breast cancer reveals enormous tumor heterogeneity yet conserved genomic imbalances and gain of MYC during progression. Am J Pathol 181, 1807-1822.

Hu, M., Yao, J., Carroll, D. K., Weremowicz, S., Chen, H., Carrasco, D., Richardson, A., Violette, S., Nikolskaya, T., Nikolsky, Y., et al. (2008). Regulation of in situ to invasive breast carcinoma transition. Cancer Cell 13, 394-406.

Hu, Y., and Smyth, G. K. (2009). ELDA: extreme limiting dilution analysis for comparing depleted and enriched populations in stem cell and other assays. J Immunol Methods 347, 70-78.

Hwang, E. S., Hyslop, T., Lynch, T., Frank, E., Pinto, D., Basila, D., Collyar, D., Bennett, A., Kaplan, C., Rosenberg, S., et al. (2019). The COMET (Comparison of Operative versus Monitoring and Endocrine Therapy) trial: a phase III randomised controlled clinical trial for low-risk ductal carcinoma in situ (DCIS). BMJ Open 9, e026797.

Hwang, E. S., and Malek, V. (2019). Estimating the magnitude of clinical benefit of local therapy in patients with DCIS. The Breast 48, S34-S38.

Jaillon, S., Ponzetta, A., Di Mitri, D., Santoni, A., Bonecchi, R., and Mantovani, A. (2020). Neutrophil diversity and plasticity in tumour progression and therapy. Nat Rev Cancer 20, 485-503.

Jolly, M. K., Somarelli, J. A., Sheth, M., Biddle, A., Tripathi, S. C., Armstrong, A. J., Hanash, S. M., Bapat, S. A., Rangarajan, A., and Levine, H. (2019). Hybrid epithelial/mesenchymal phenotypes promote metastasis and therapy resistance across carcinomas. Pharmacol Ther 194, 161-184.

Kanbayashi, C., and Iwata, H. (2017). Current approach and future perspective for ductal carcinoma in situ of the breast. Jpn J Clin Oncol 47, 671-677.

Katzenelenbogen, Y., Sheban, F., Yalin, A., Yofe, I., Svetlichnyy, D., Jaitin, D. A., Bornstein, C., Moshe, A., Keren-Shaul, H., Cohen, M., et al. (2020). Coupled scRNA-Seq and Intracellular Protein Activity Reveal an Immunosuppressive Role of TREM2 in Cancer. Cell 182, 872-885 e819.

Kong, D., Hughes, C. J., and Ford, H. L. (2020). Cellular Plasticity in Breast Cancer Progression and Therapy. Front Mol Biosci 7, 72 .

Koren, S., Reavie, L., Couto, J. P., De Silva, D., Stadler, M. B., Roloff, T., Britschgi, A., Eichlisberger, T., Kohler, H., Aina, O., et al. (2015). PIK3CA(H1047R) induces multipotency and multi-lineage mammary tumours. Nature $525,114-118$.

Kotler, B. P., and Brown, J. S. (2020). Cancer Community Ecology. Cancer Control 27.

Kroger, C., Afeyan, A., Mraz, J., Eaton, E. N., Reinhardt, F., Khodor, Y. L., Thiru, P., Bierie, B., Ye, X., Burge, C. B., and Weinberg, R. A. (2019). Acquisition of a hybrid E/M state is essential for tumorigenicity of basal breast cancer cells. Proc Natl Acad Sci U S A 116, 7353-7362. 
Lee, S., Stewart, S., Nagtegaal, I., Luo, J., Wu, Y., Colditz, G., Medina, D., and Allred, D. C. (2012).

Differentially expressed genes regulating the progression of ductal carcinoma in situ to invasive breast cancer. Cancer Res 72, 4574-4586.

Lehman, C. D., Arao, R. F., Sprague, B. L., Lee, J. M., Buist, D. S., Kerlikowske, K., Henderson, L. M., Onega, T., Tosteson, A. N., Rauscher, G. H., and Miglioretti, D. L. (2017). National Performance Benchmarks for Modern Screening Digital Mammography: Update from the Breast Cancer Surveillance Consortium. Radiology 283, 49-58.

Li, H., Handsaker, B., Wysoker, A., Fennell, T., Ruan, J., Homer, N., Marth, G., Abecasis, G., Durbin, R., and Proc, G. P. D. (2009). The Sequence Alignment/Map format and SAMtools. Bioinformatics 25, 2078-2079.

Lilja, A. M., Rodilla, V., Huyghe, M., Hannezo, E., Landragin, C., Renaud, O., Leroy, O., Rulands, S., Simons, B. D., and Fre, S. (2018). Clonal analysis of Notch1-expressing cells reveals the existence of unipotent stem cells that retain long-term plasticity in the embryonic mammary gland. Nat Cell Biol 20,677-687.

Lim, E., Vaillant, F., Wu, D., Forrest, N. C., Pal, B., Hart, A. H., Asselin-Labat, M. L., Gyorki, D. E., Ward, T., Partanen, A., et al. (2009). Aberrant luminal progenitors as the candidate target population for basal tumor development in BRCA1 mutation carriers. Nat Med 15, 907-913.

Love, M. I., Huber, W., and Anders, S. (2014). Moderated estimation of fold change and dispersion for RNA-seq data with DESeq2. Genome Biol 15, 550.

Ma, X. J., Salunga, R., Tuggle, J. T., Gaudet, J., Enright, E., McQuary, P., Payette, T., Pistone, M., Stecker, K., Zhang, B. M., et al. (2003). Gene expression profiles of human breast cancer progression. Proc Natl Acad Sci U S A 100, 5974-5979.

Mani, S. A., Guo, W., Liao, M. J., Eaton, E. N., Ayyanan, A., Zhou, A. Y., Brooks, M., Reinhard, F., Zhang, C. C., Shipitsin, M., et al. (2008). The epithelial-mesenchymal transition generates cells with properties of stem cells. Cell 133, 704-715.

McAllister, S. S., and Weinberg, R. A. (2014). The tumour-induced systemic environment as a critical regulator of cancer progression and metastasis. Nat Cell Biol 16, 717-727.

McFarlane, R. J., and Wakeman, J. A. (2017). Meiosis-like Functions in Oncogenesis: A New View of Cancer. Cancer Res 77, 5712-5716.

McGinnis, C. S., Murrow, L. M., and Gartner, Z. J. (2019). DoubletFinder: Doublet Detection in Single-Cell RNA Sequencing Data Using Artificial Nearest Neighbors. Cell Syst 8, 329-337 e324.

Meyer, D. S., Brinkhaus, H., Muller, U., Muller, M., Cardiff, R. D., and Bentires-Alj, M. (2011). Luminal expression of PIK3CA mutant H1047R in the mammary gland induces heterogeneous tumors. Cancer Res 71, 4344-4351. 
Mishalian, I., Bayuh, R., Levy, L., Zolotarov, L., Michaeli, J., and Fridlender, Z. G. (2013). Tumor-associated neutrophils (TAN) develop pro-tumorigenic properties during tumor progression. Cancer Immunol Immunother 62, 1745-1756.

Molgora, M., Esaulova, E., Vermi, W., Hou, J., Chen, Y., Luo, J., Brioschi, S., Bugatti, M., Omodei, A. S., Ricci, B., et al. (2020). TREM2 Modulation Remodels the Tumor Myeloid Landscape Enhancing Anti-PD-1 Immunotherapy. Cell 182, 886-900 e817.

Molyneux, G., Geyer, F. C., Magnay, F.-A., McCarthy, A., Kendrick, H., Natrajan, R., MacKay, A., Grigoriadis, A., Tutt, A., Ashworth, A., et al. (2010). BRCA1 Basal-like Breast Cancers Originate from Luminal Epithelial Progenitors and Not from Basal Stem Cells. Cell Stem Cell 7, 403-417.

Nelson, A. C., Machado, H. L., and Schwertfeger, K. L. (2018). Breaking through to the Other Side: Microenvironment Contributions to DCIS Initiation and Progression. J Mammary Gland Biol Neoplasia 23, 207-221.

Nguyen, Q. H., Pervolarakis, N., Blake, K., Ma, D., Davis, R. T., James, N., Phung, A. T., Willey, E., Kumar, R., Jabart, E., et al. (2018). Profiling human breast epithelial cells using single cell RNA sequencing identifies cell diversity. Nat Commun 9, 2028.

Nieto, M. A., Huang, R. Y., Jackson, R. A., and Thiery, J. P. (2016). Emt: 2016. Cell 166, 21-45.

Ostrand-Rosenberg, S., and Fenselau, C. (2018). Myeloid-Derived Suppressor Cells: Immune-Suppressive Cells That Impair Antitumor Immunity and Are Sculpted by Their Environment. J Immunol 200, $422-431$.

Pal, B., Chen, Y., Vaillant, F., Jamieson, P., Gordon, L., Rios, A. C., Wilcox, S., Fu, N., Liu, K. H., Jackling, F. C., et al. (2017). Construction of developmental lineage relationships in the mouse mammary gland by single-cell RNA profiling. Nat Commun 8, 1627.

Pinder, S. E. (2010). Ductal carcinoma in situ (DCIS): pathological features, differential diagnosis, prognostic factors and specimen evaluation. Mod Pathol 23 Suppl 2, S8-13.

Qiu, X., Hill, A., Packer, J., Lin, D., Ma, Y. A., and Trapnell, C. (2017a). Single-cell mRNA quantification and differential analysis with Census. Nat Methods 14, 309-315.

Qiu, X., Mao, Q., Tang, Y., Wang, L., Chawla, R., Pliner, H. A., and Trapnell, C. (2017b). Reversed graph embedding resolves complex single-cell trajectories. Nat Methods 14, 979-982.

Quail, D. F., and Joyce, J. A. (2013). Microenvironmental regulation of tumor progression and metastasis. Nat Med 19, 1423-1437.

Regan, J. L., and Smalley, M. J. (2020). Integrating single-cell RNA-sequencing and functional assays to decipher mammary cell states and lineage hierarchies. NPJ Breast Cancer 6, 32. 
Ribatti, D., Tamma, R., and Annese, T. (2020). Epithelial-Mesenchymal Transition in Cancer: A Historical Overview. Transl Oncol 13, 100773.

Risom, T., Langer, E. M., Chapman, M. P., Rantala, J., Fields, A. J., Boniface, C., Alvarez, M. J., Kendsersky, N. D., Pelz, C. R., Johnson-Camacho, K., et al. (2018). Differentiation-state plasticity is a targetable resistance mechanism in basal-like breast cancer. Nat Commun 9, 3815.

Rosenthal, A. Z., Qi, Y., Hormoz, S., Park, J., Li, S. H., and Elowitz, M. B. (2018). Metabolic interactions between dynamic bacterial subpopulations. Elife 7 .

Schneider, C. A., Rasband, W. S., and Eliceiri, K. W. (2012). NIH Image to ImageJ: 25 years of image analysis. Nat Methods 9, 671-675.

Seth, A., Kitching, R., Landberg, G., Xu, J., Zubovits, J., and Burger, A. M. (2003). Gene expression profiling of ductal carcinomas in situ and invasive breast tumors. Anticancer Res 23, 2043-2051.

Siegel, R. L., Miller, K. D., and Jemal, A. (2018). Cancer statistics, 2018. CA Cancer J Clin 68, 7-30.

Silverstein, M. J., and Lagios, M. D. (2014). Ductal Carcinoma In Situ of the Breast. In Breast cancer : a new era in management, D.S. Francescatti, and M.J. Silverstein, eds. (Springer), pp. 269-311.

Sinha, V. C., and Piwnica-Worms, H. (2018). Intratumoral Heterogeneity in Ductal Carcinoma In Situ: Chaos and Consequence. J Mammary Gland Biol Neoplasia 23, 191-205.

Siwko, S. K., Bu, W., Gutierrez, C., Lewis, B., Jechlinger, M., Schaffhausen, B., and Li, Y. (2008). Lentivirusmediated oncogene introduction into mammary cells in vivo induces tumors. Neoplasia 10,653-U651.

Stuart, T., Butler, A., Hoffman, P., Hafemeister, C., Papalexi, E., Mauck, W. M., 3rd, Hao, Y., Stoeckius, M., Smibert, P., and Satija, R. (2019). Comprehensive Integration of Single-Cell Data. Cell 177, 1888-1902 e1821.

Sun, Z., Wang, C.-Y., Lawson, D. A., Kwek, S., Velozo, H. G., Owyong, M., Lai, M.-D., Fong, L., Wilson, M., Su, $\mathrm{H}$. , et al. (2017). Single-cell RNA sequencing reveals gene expression signatures of breast cancerassociated endothelial cells. Oncotarget 9, 10945-10961.

Thong, T., Wang, Y., Brooks, M. D., Lee, C. T., Scott, C., Balzano, L., Wicha, M. S., and Colacino, J. A. (2020). Hybrid Stem Cell States: Insights Into the Relationship Between Mammary Development and Breast Cancer Using Single-Cell Transcriptomics. Front Cell Dev Biol 8, 288.

Tirosh, I., Izar, B., Prakadan, S. M., Wadsworth, M. H., 2nd, Treacy, D., Trombetta, J. J., Rotem, A., Rodman, C., Lian, C., Murphy, G., et al. (2016). Dissecting the multicellular ecosystem of metastatic melanoma by single-cell RNA-seq. Science (1979) 352, 189-196. 
Trapnell, C., Cacchiarelli, D., Grimsby, J., Pokharel, P., Li, S., Morse, M., Lennon, N. J., Livak, K. J., Mikkelsen, T. S., and Rinn, J. L. (2014). The dynamics and regulators of cell fate decisions are revealed by pseudotemporal ordering of single cells. Nat Biotechnol 32, 381-386.

Van Keymeulen, A., Lee, M. Y., Ousset, M., Brohee, S., Rorive, S., Giraddi, R. R., Wuidart, A., Bouvencourt, G., Dubois, C., Salmon, I., et al. (2015). Reactivation of multipotency by oncogenic PIK3CA induces breast tumour heterogeneity. Nature 525, 119-123.

van Seijen, M., Lips, E. H., Thompson, A. M., Nik-Zainal, S., Futreal, A., Hwang, E. S., Verschuur, E., Lane, J., Jonkers, J., Rea, D. W., and Wesseling, J. (2019). Ductal carcinoma in situ: to treat or not to treat, that is the question. Br J Cancer 121, 285-292.

Veglia, F., Perego, M., and Gabrilovich, D. (2018). Myeloid-derived suppressor cells coming of age. Nat Immunol 19, 108-119.

Vincent-Salomon, A., Lucchesi, C., Gruel, N., Raynal, V., Pierron, G., Goudefroye, R., Reyal, F., Radvanyi, F., Salmon, R., Thiery, J. P., et al. (2008). Integrated genomic and transcriptomic analysis of ductal carcinoma in situ of the breast. Clin Cancer Res 14, 1956-1965.

Wagner, J., Rapsomaniki, M. A., Chevrier, S., Anzeneder, T., Langwieder, C., Dykgers, A., Rees, M., Ramaswamy, A., Muenst, S., Soysal, S. D., et al. (2019). A Single-Cell Atlas of the Tumor and Immune Ecosystem of Human Breast Cancer. Cell 177, 1330-1345 e1318.

Wahl, G. M., and Spike, B. T. (2017). Cell state plasticity, stem cells, EMT, and the generation of intratumoral heterogeneity. NPJ Breast Cancer 3,14.

Wei, S. C., Levine, J. H., Cogdill, A. P., Zhao, Y., Anang, N. A. S., Andrews, M. C., Sharma, P., Wang, J., Wargo, J. A., Pe'er, D., and Allison, J. P. (2017). Distinct Cellular Mechanisms Underlie Anti-CTLA-4 and Anti-PD-1 Checkpoint Blockade. Cell 170, 1120-1133 e1117.

Weigelt, B., Eberle, C., Cowell, C. F., Ng, C. K., and Reis-Filho, J. S. (2014). Metaplastic breast carcinoma: more than a special type. Nat Rev Cancer 14, 147-148.

Welte, T., Kim, I. S., Tian, L., Gao, X., Wang, H., Li, J., Holdman, X. B., Herschkowitz, J. I., Pond, A., Xie, G., et al. (2016). Oncogenic mTOR signalling recruits myeloid-derived suppressor cells to promote tumour initiation. Nat Cell Biol 18,632-644.

Wu, L., Awaji, M., Saxena, S., Varney, M. L., Sharma, B., and Singh, R. K. (2020). IL-17-CXC Chemokine Receptor 2 Axis Facilitates Breast Cancer Progression by Up-Regulating Neutrophil Recruitment. Am J Pathol 190, 222-233.

Wuidart, A., Sifrim, A., Fioramonti, M., Matsumura, S., Brisebarre, A., Brown, D., Centonze, A., Dannau, A., Dubois, C., Van Keymeulen, A., et al. (2018). Early lineage segregation of multipotent embryonic mammary gland progenitors. Nat Cell Biol 20,666-676. 
Yang, J., Antin, P., Berx, G., Blanpain, C., Brabletz, T., Bronner, M., Campbell, K., Cano, A., Casanova, J., Christofori, G., et al. (2020). Guidelines and definitions for research on epithelial-mesenchymal transition. Nat Rev Mol Cell Biol 21,341-352.

Yeh, I. T., and Mies, C. (2008). Application of immunohistochemistry to breast lesions. Arch Pathol Lab Med 132, 349-358.

Yeo, S. K., Zhu, X., Okamoto, T., Hao, M., Wang, C., Lu, P., Lu, L. J., and Guan, J. L. (2020). Single-cell RNAsequencing reveals distinct patterns of cell state heterogeneity in mouse models of breast cancer. Elife 9 .

Zhang, J., Gurusaran, M., Fujiwara, Y., Zhang, K., Echbarthi, M., Vorontsov, E., Guo, R., Pendlebury, D. F., Alam, I., Livera, G., et al. (2020). The BRCA2-MEILB2-BRME1 complex governs meiotic recombination and impairs the mitotic BRCA2-RAD51 function in cancer cells. Nat Commun 11, 2055.

Zhang, Q., Liu, S., Ge, D., Zhang, Q., Xue, Y., Xiong, Z., Abdel-Mageed, A. B., Myers, L., Hill, S. M., Rowan, B. G., et al. (2012). Interleukin-17 promotes formation and growth of prostate adenocarcinoma in mouse models. Cancer Res 72, 2589-2599.

Zhang, Y., Zoltan, M., Riquelme, E., Xu, H., Sahin, I., Castro-Pando, S., Montiel, M. F., Chang, K., Jiang, Z., Ling, J., et al. (2018). Immune Cell Production of Interleukin 17 Induces Stem Cell Features of Pancreatic Intraepithelial Neoplasia Cells. Gastroenterology 155, 210-223 e213.

Zhao, F., Hoechst, B., Duffy, A., Gamrekelashvili, J., Fioravanti, S., Manns, M. P., Greten, T. F., and Korangy, F. (2012). S100A9 a new marker for monocytic human myeloid-derived suppressor cells. Immunology $136,176-183$.

Zheng, G. X., Terry, J. M., Belgrader, P., Ryvkin, P., Bent, Z. W., Wilson, R., Ziraldo, S. B., Wheeler, T. D., McDermott, G. P., Zhu, J., et al. (2017). Massively parallel digital transcriptional profiling of single cells. Nat Commun 8, 14049.

\section{Figures}


A

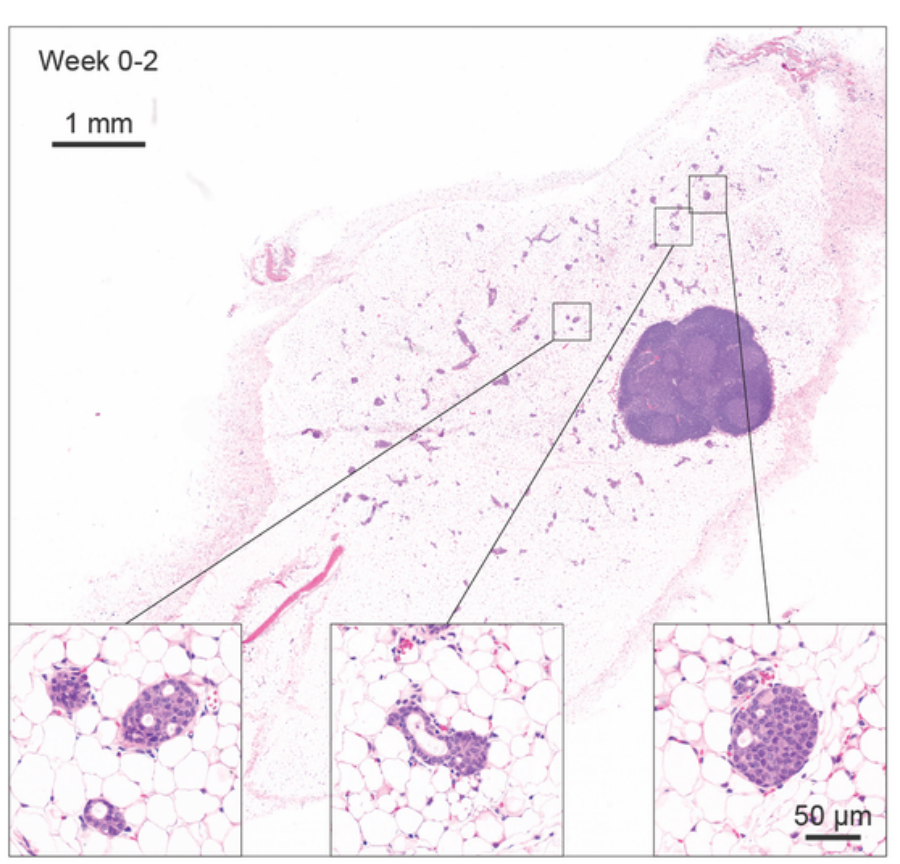

C

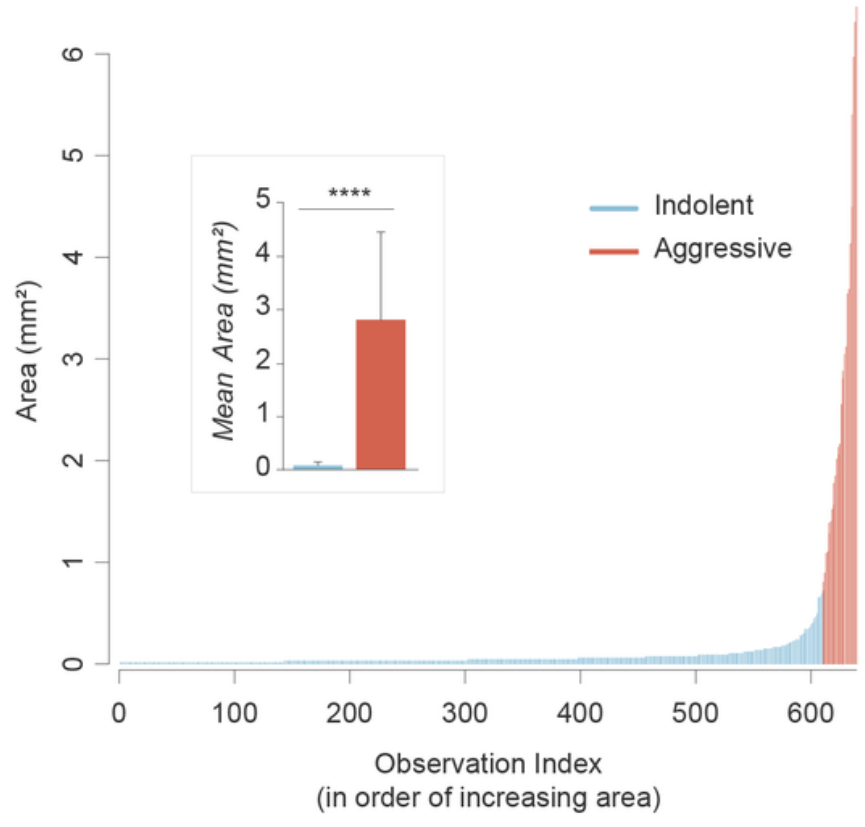

B

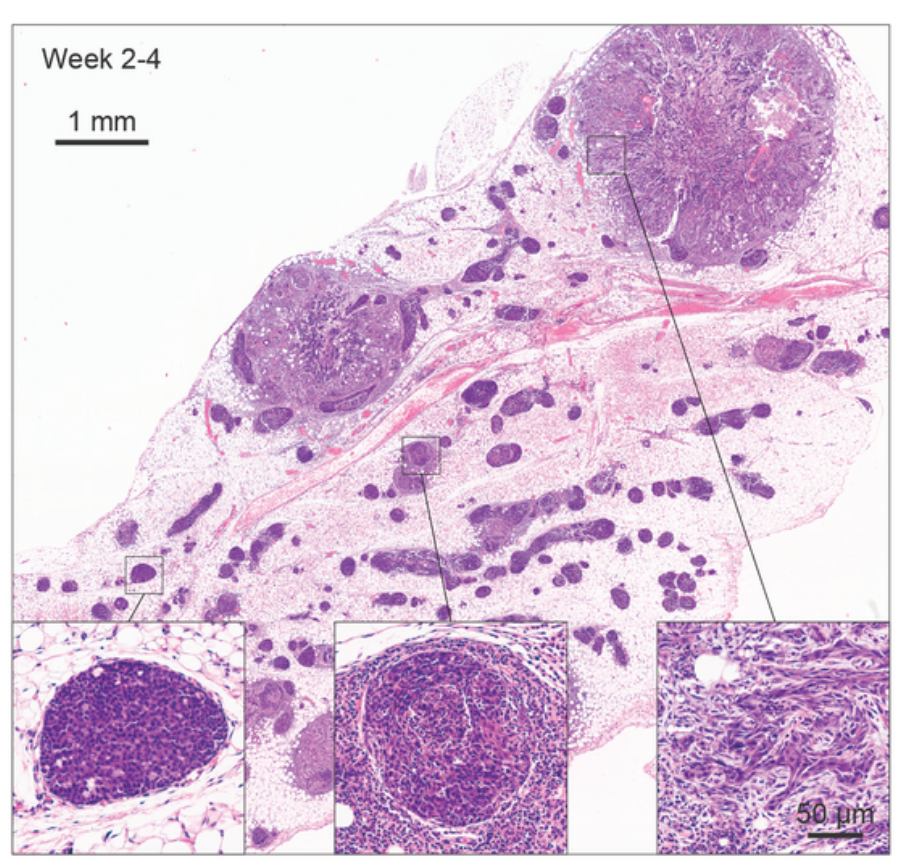

D
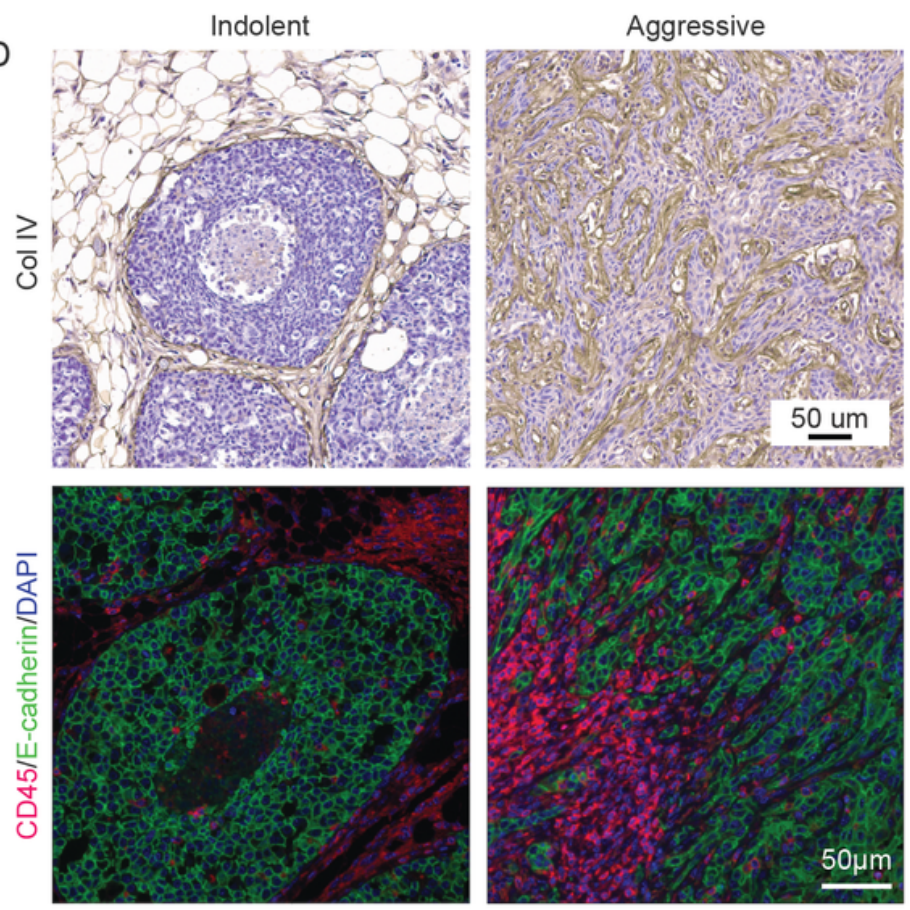

\section{Figure 1}

Aggressive progression occurs in a minority of breast lesions and is associated with heavy immune infiltration (A, B) Mouse model of breast cancer exhibits progression of mammary lesions over time. Mammary glands were collected from animals at various time points following intraductal injection and stained by hematoxylin \& eosin (H\&E) to visualize lesions at 0-2 weeks (A) or 2-4 weeks (B) after injection. High-power magnification (inset) shows in situ lesions of early time points (A), and a combination of in situ (indolent; B, left inset) and invasive (aggressive; B, right inset) lesions at later time points. Lesions exhibiting a well-circumscribed tumor compartment with heavy stromal involvement (B, center inset) were 
also observed. (C) Quantification of lesions present 2-4 weeks after injection reveals minority of rapidlyprogressing large lesions. Mammary glands bearing a combination of indolent and aggressive lesions were evaluated for individual lesion size. Index plot shows all lesions quantified across 5 animals. Inset bar graph shows mean area of top $5 \%$ largest lesions versus remainder. P-value $(<2.2 \mathrm{e}-16)$ was calculated using two-sided Wilcoxon rank sum test for non-normal data; error bars indicate standard deviation (S.D.), $U=0$. (D) Aggressive lesions are heavily immune infiltrated. Lesion-bearing glands were immunostained for collagen IV (top panel) and E-cadherin/CD45 (bottom panel) to assess, respectively, integrity of basement membrane and associated immune infiltration of indolent and aggressive lesions. 
A

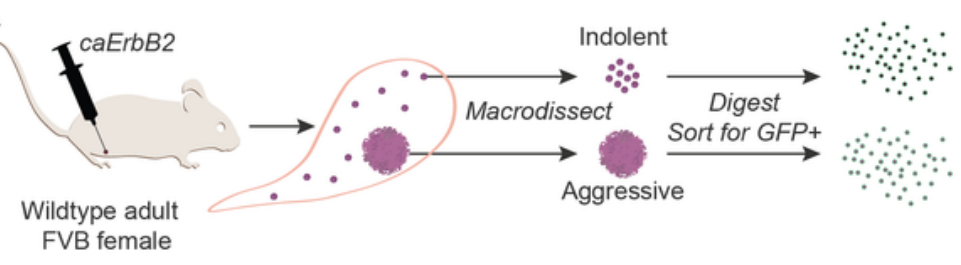

B
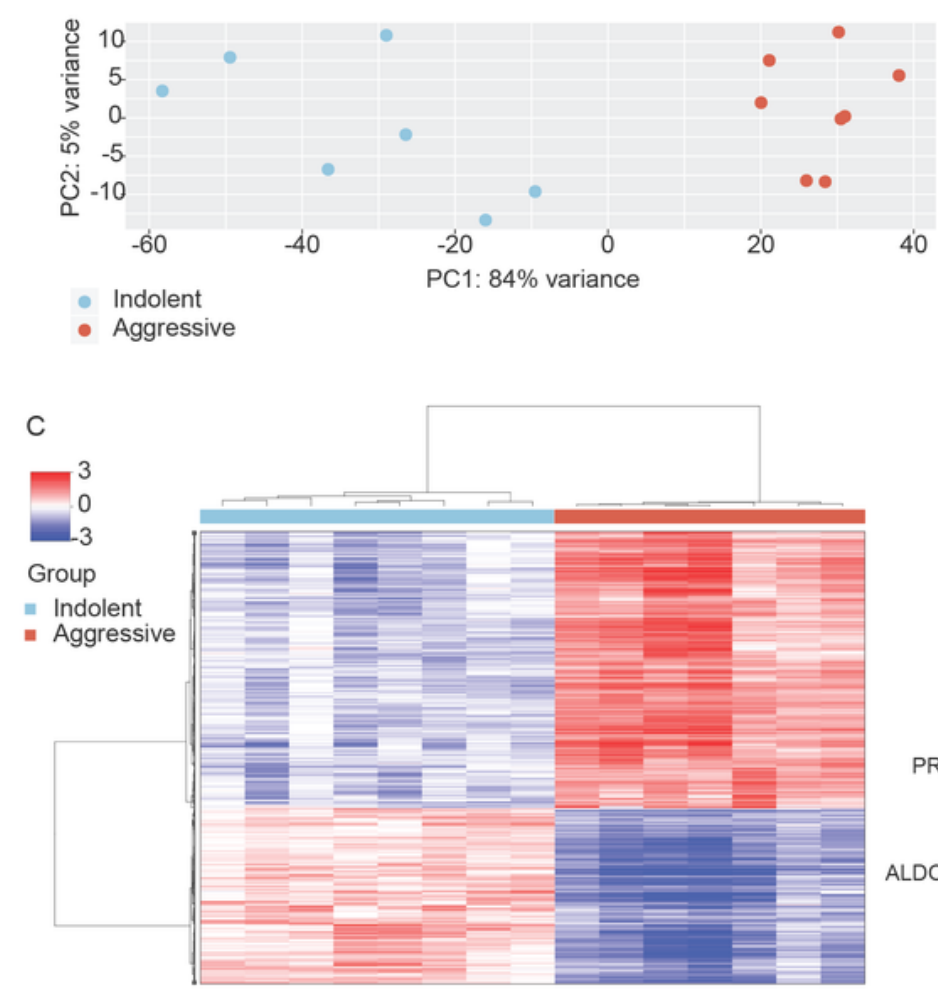

CITRATE CYCLE TCA CYCLE PROGESTERONE_MEDIATED_OOCYTE_MATURATION ARGININE_AND_PROLINE_METABOLISM AXON_GUIDANCE BLADDER CANCER ALDOSTERONE_REGULATED_SODIUM_REABSORPTION •

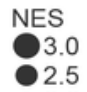

2.5 Upregulated in Aggressive

- 2.0 Downregulated in Aggressive
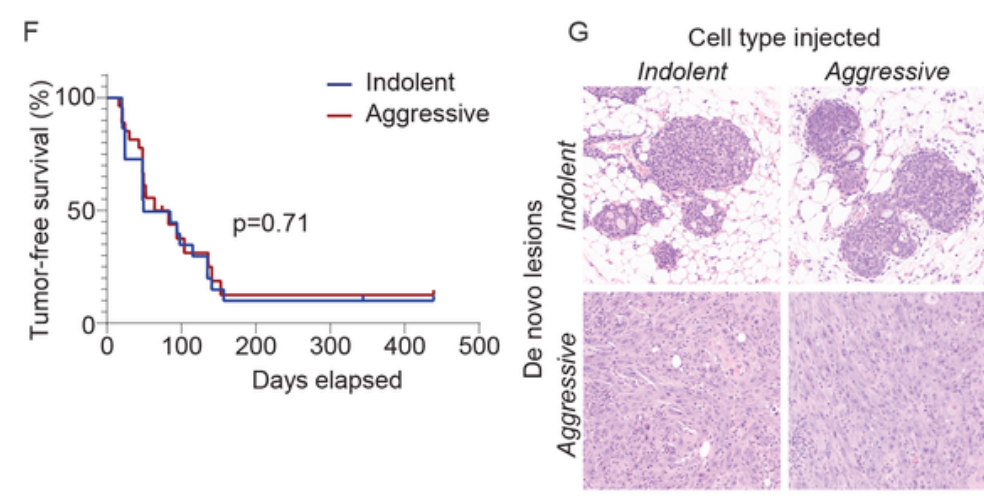

\section{Figure 2}

Tumor cells from indolent versus aggressive lesions are transcriptionally distinct, but functionally indistinguishable in a new host (A) Schema of bulk RNA sequencing assay. Lesion samples were pooled from 2-5 animals per sample (21 total animals) to acquire sufficient material for analysis. (B) Principle component analysis of indolent versus aggressive samples. (C) Heatmap of differentially expressed (DE) genes (FDR <0.05) in indolent versus aggressive samples. Values indicate median-centered variance- 
stabilizing-transformed counts. (D, E) Normalized enrichment scores of Hallmarks (D) and KEGG (E) pathways in aggressive lesions versus indolent following gene set enrichment analysis (GSEA).

Pathways with FDR $<0.05$ shown in order of normalized enrichment score (NES). Red and blue NES indicate, respectively, positive and negative enrichment in aggressive samples. (F) Kaplan-Meier plot showing tumor-free survival curves of animals injected intraductally with indolent or aggressive tumor cells. P-value calculated using log-rank (Mantel-Cox) test. Indolent group, $\mathrm{n}=22$; aggressive group, $\mathrm{n}=27$. (G) H\&E of de novo lesions arising in the host after intraductal injection of indolent or aggressive tumor cells. $(H)$ Proportion of animals bearing tumors or remaining tumor-free $\sim 400$ days following injection of limiting dilutions of indolent or aggressive tumor cells. Number evaluated per group and dilution indicated in pie. Estimated tumor initiating cell frequency (Est. TIC freq.) calculated using Extreme Limiting Dilution Analysis. NS, non-significant, $p$ value $>0.05$. 


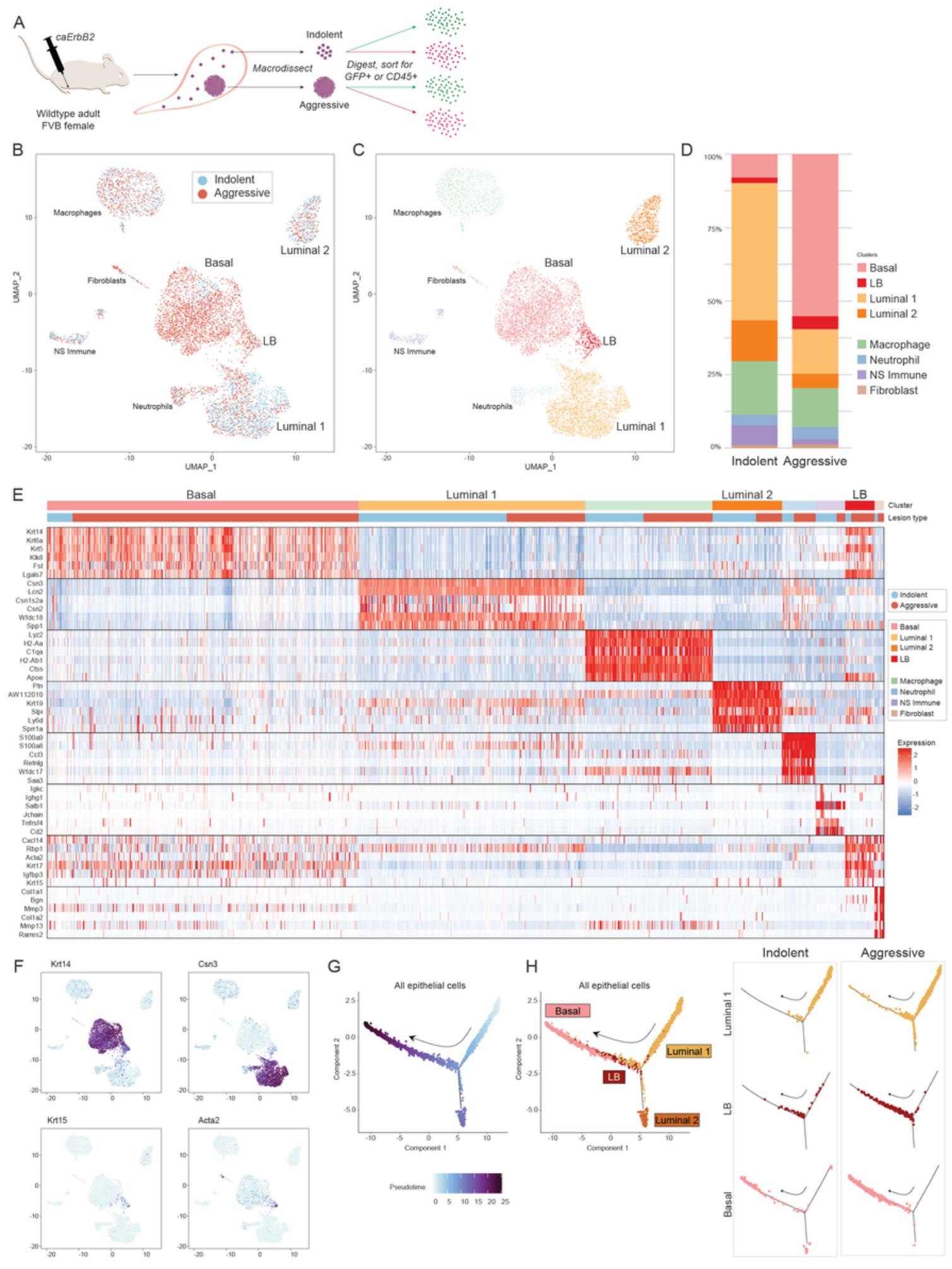

\section{Figure 3}

The aggressive tumor niche harbors an expanded basal-like population that is transcriptionally linked to a luminal-like population through an intermediate state (A) Schema of single-cell RNA sequencing assay. Four samples (indolent tumor cells, indolent immune cells, aggressive tumor cells, and aggressive immune cells) were obtained from 13 pooled mice to acquire sufficient material for analysis. (B-D) Uniform Manifold Approximation and Projection (UMAP) was used for dimension reduction of single-cell 
data. Tumor fractions from indolent and aggressive lesions were computationally merged (B) for cluster analysis and identification (C). Proportion of clusters per group shown in (D). (E) Heatmap of top differentially-expressed (DE) genes for each cluster, ordered in decreasing cluster size. Clusters indicated by top horizontal bar, with inferred tumor clusters noted (Basal, Luminal 1, Luminal 2, LB). Cells derived from indolent or aggressive tumor sample indicated by second horizontal bar. DE genes are listed on the left. (F) UMAP plots of all tumor cells, colored by expression of Krt14 for basal-like cells, Csn3 for Luminal 1 cells, Krt15 and Acta2 for LB cells. (G, H) Trajectory plot of all tumor cells, colored by pseudotime value (G) and cluster ( $\mathrm{H}$, left panel). Luminal 1, LB, and Basal cells were plotted by lesion type to visualize distribution along trajectory of cells from indolent versus aggressive tumors $(\mathrm{H}$, right panel). Arrow indicates increasing pseudotime value. 
Indolent

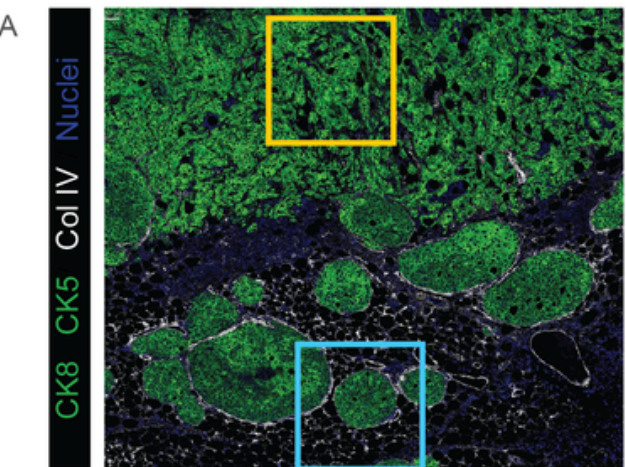

B

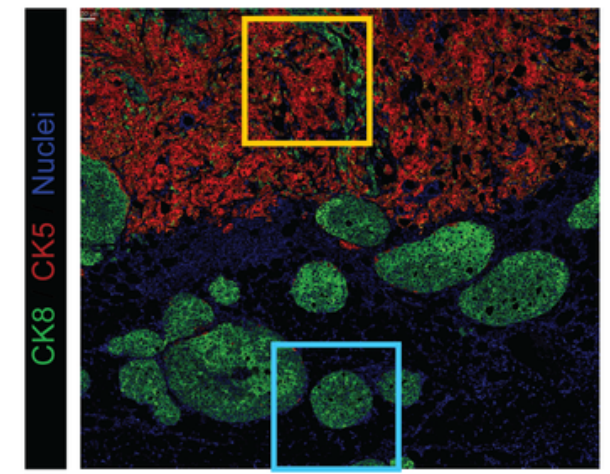

C

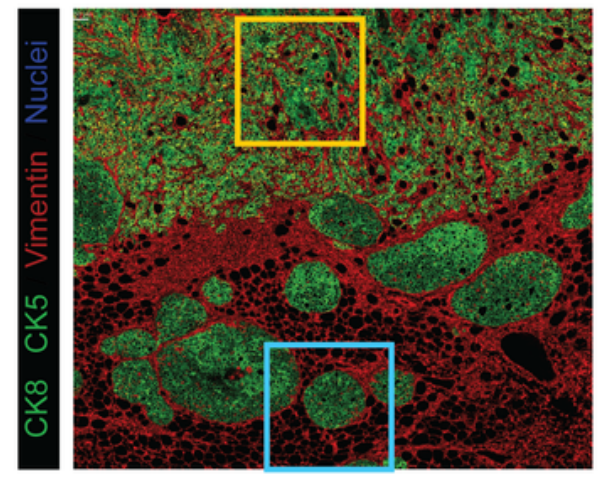

D

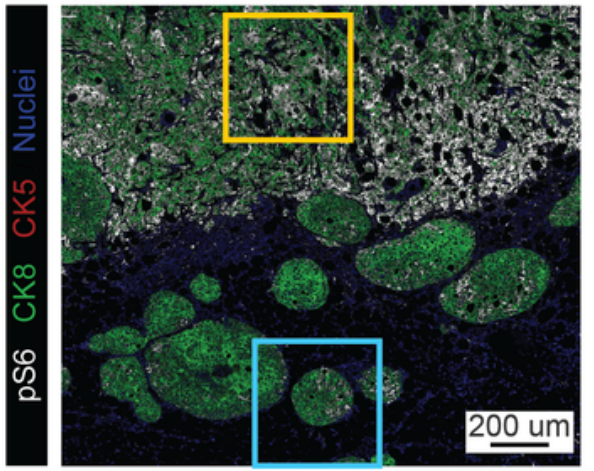

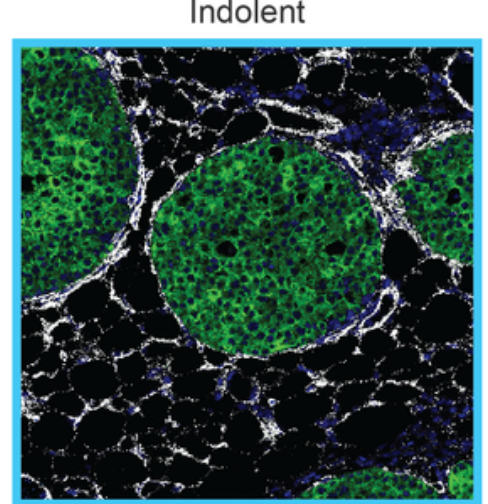
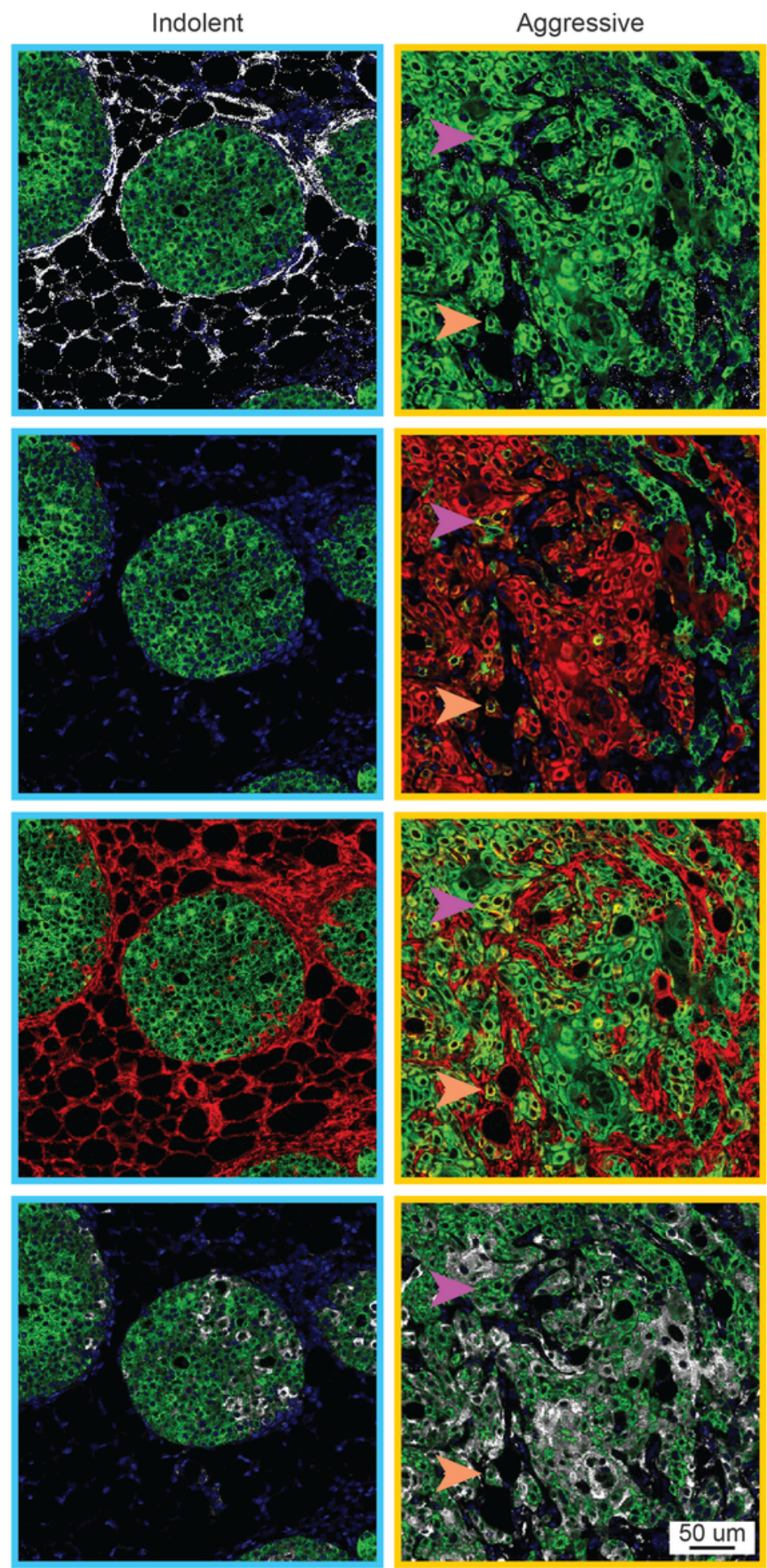

Figure 4

Multi-parametric imaging of indolent and aggressive lesions reveals spatially heterogeneous tumor and immune niches (A-D) All images shown are pseudo-colored channels from a single mammary tissue section bearing indolent and aggressive lesions stained using a cocktail of antibodies and visualized using imaging mass cytometry. Lesions were stained for collagen IV (basement membrane), cytokeratin 8 (luminal epithelial marker), cytokeratin 5 (basal epithelial marker), vimentin (mesenchymal marker), and 
pS6 (S235/S236, mTOR signaling). Combinations of markers were selected to evaluate indolent and aggressive lesions for membrane integrity (A), epithelial identity and mixed epithelial lineage (B), mixed epithelial/mesenchymal lineage (C), and metabolic activity (D). Representative images on the left panel show low-magnification view of adjacent indolent and aggressive lesions. Boxes in left panel correspond by color to high magnification images, shown in middle (blue, indolent) and right (yellow, aggressive) panels. Arrows of the same color point to the same cell in each image.
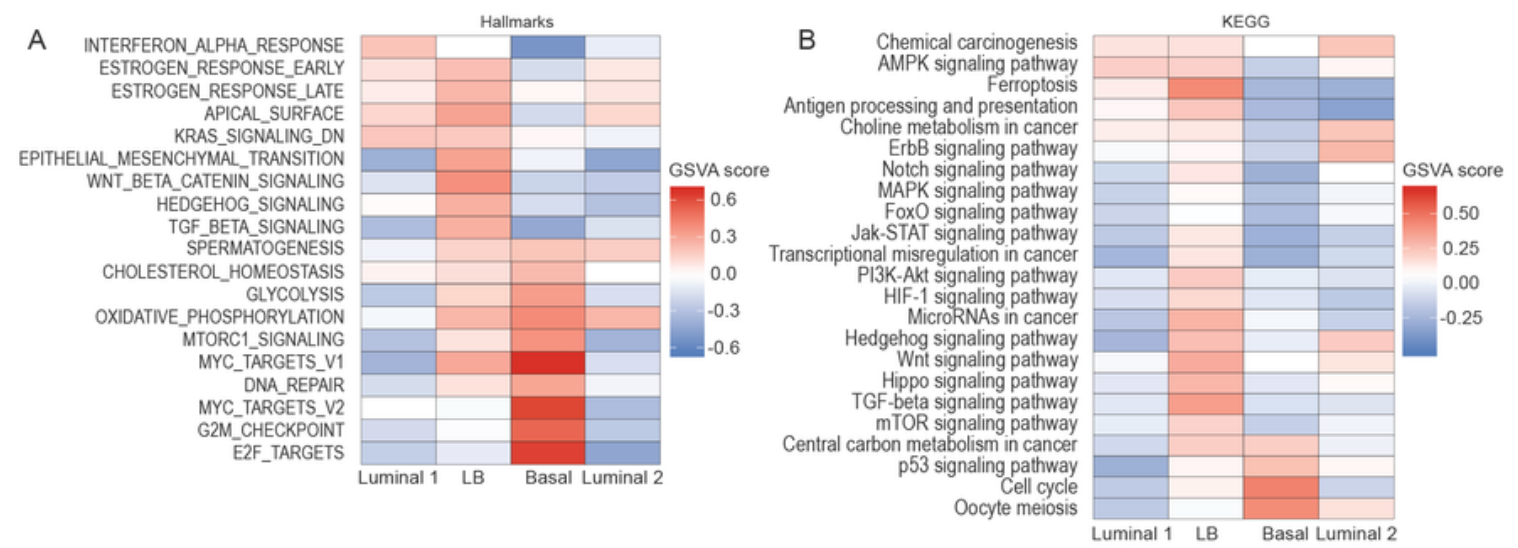

C

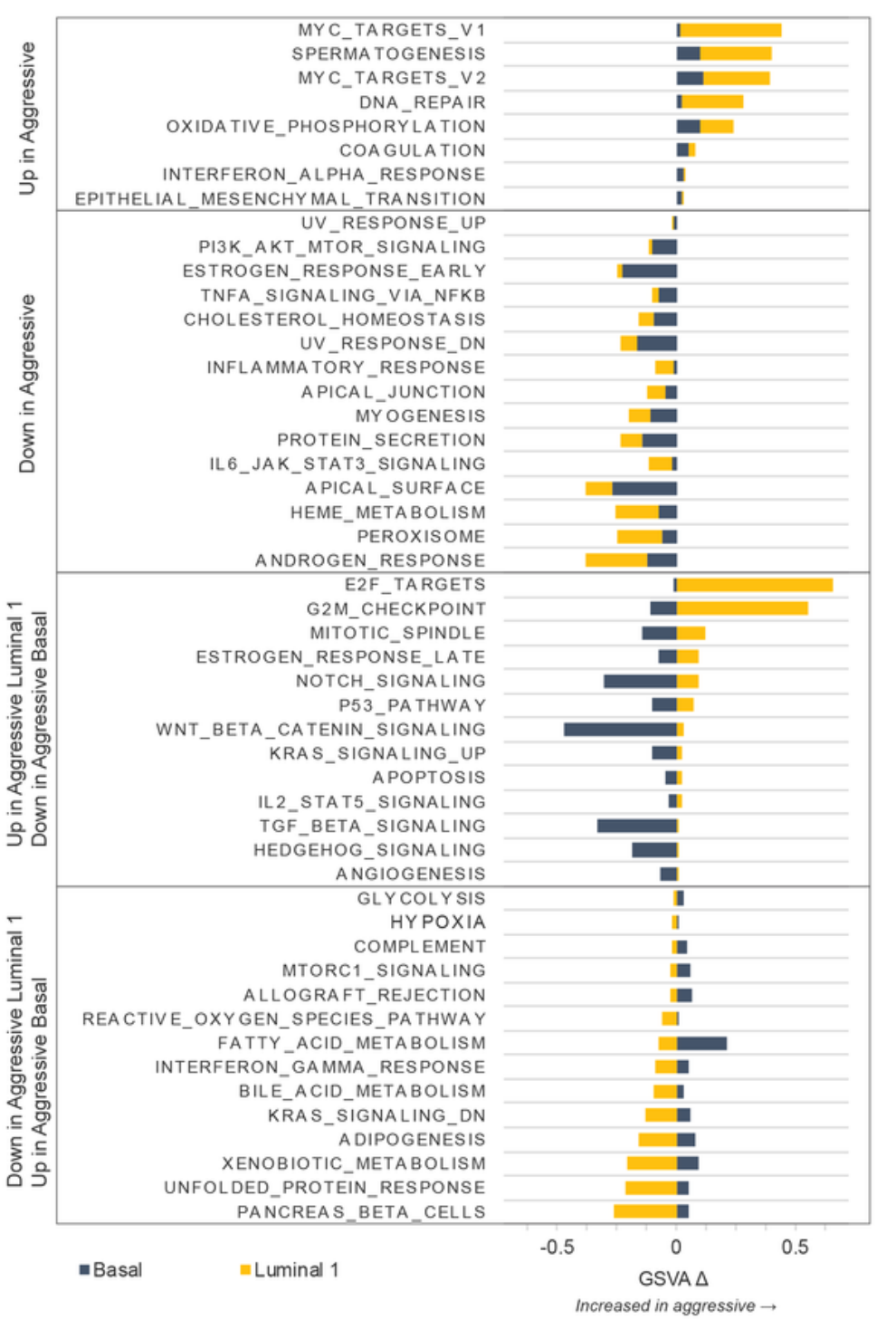

Figure 5 
Heterogeneous pathway enrichment across tumor subpopulations suggest distinct functional roles in the tumor niche (A, B) Comparison of pathway enrichment across tumor subpopulations. Heatmap of enrichment scores of selected Hallmarks (A) and KEGG (B) pathways in tumor clusters following gene set variation analysis (GSVA). Positive score indicates relative enrichment versus other populations identified by scRNA-seq and pathways within the same collection. Cells from indolent and aggressive lesions were analyzed together per cluster. (C) Comparison of pathway changes in indolent versus aggressive lesions: Clusters were analyzed by lesion type to calculate a change in Hallmark pathway enrichment score (GSVA delta, $\Delta)$ in aggressive versus indolent lesions. Positive score indicates increased score in aggressive lesions. Pathways were classified as concordantly (top two panels) or discordantly (bottom two panels) shifted in Luminal 1 and Basal populations of aggressive lesions compared to indolent lesions. 

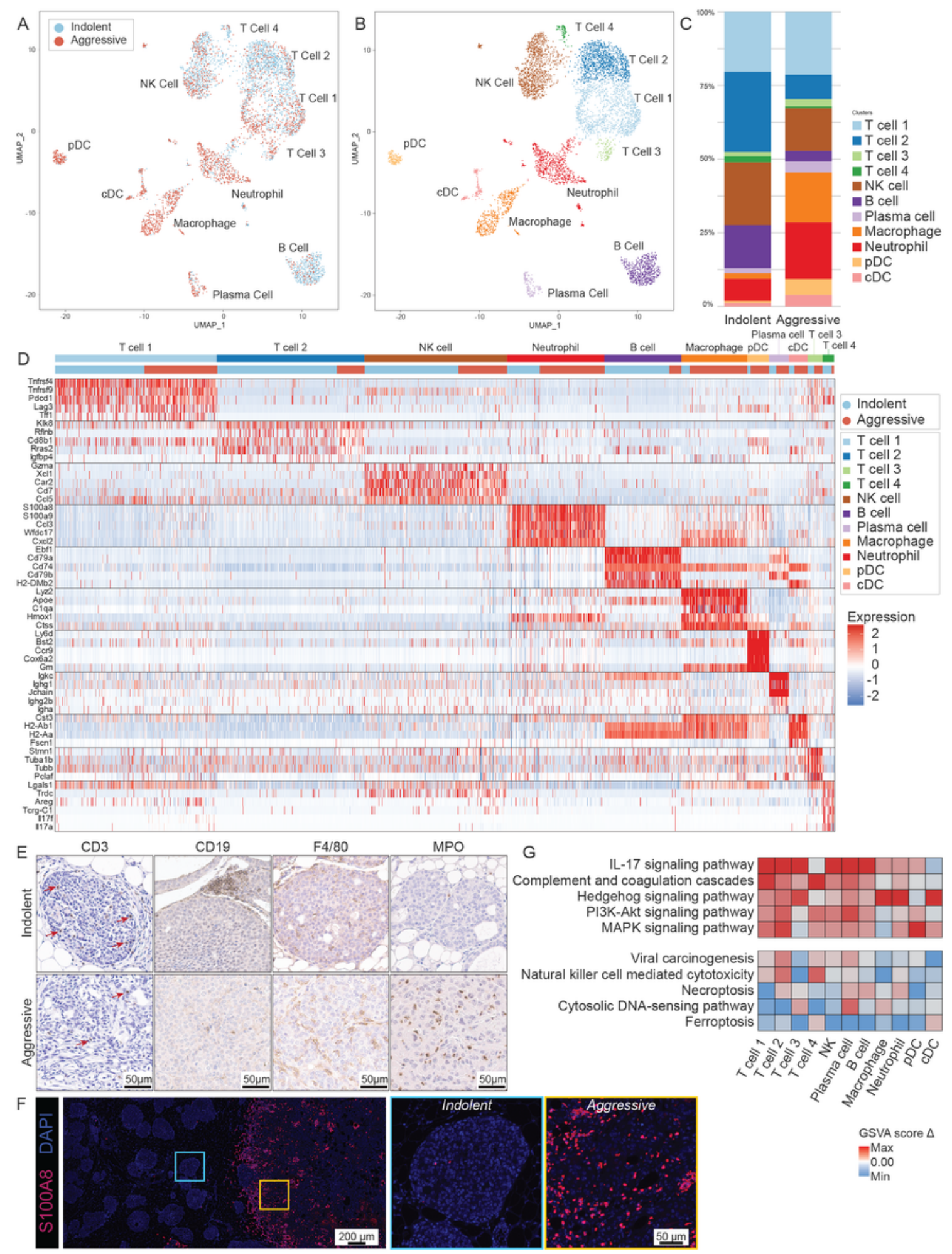
Ferroptosis

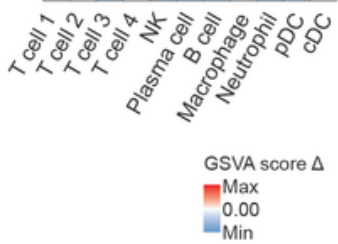

\section{Figure 6}

The aggressive niche harbors an expanded and spatially-restricted myeloid population (A-C) UMAP was used for dimension reduction of single-cell data. Immune fractions from indolent and aggressive lesions were computationally merged (A) for cluster analysis and identification (B). Proportion of clusters per group shown in (C). NK - natural killer; pDC - plasmacytoid dendritic cell; CDC - conventional dendritic cell (D) Heatmap of top differentially-expressed (DE) genes for each cluster, ordered in decreasing cluster 
size. Clusters indicated by top horizontal bar, with inferred immune identity noted. Cells derived from indolent or aggressive tumor sample indicated by second horizontal bar. DE genes are listed on the left. (E) Immunohistochemical staining for major lymphoid (T cells - CD3; B cells - CD19) and myeloid (macrophages - F4/80; neutrophils - MPO) populations associated with indolent and aggressive lesions. Arrows point to selected positively stained cells. Representative images shown. (F) Immunofluorescent staining for neutrophils using an alternative marker S100A8. Left panel shows low power magnification view of adjacent indolent and aggressive lesions, with S100A8+ infiltration tightly localized with the aggressive niche. Boxes correspond by color to high magnification images shown middle (blue, indolent) and right (yellow, aggressive) panels. Representative images shown. (G) Immune clusters were analyzed by lesion type to calculate a change in KEGG pathway enrichment score (GSVA delta) in aggressive versus indolent lesions. Positive score indicates increased enrichment score in aggressive lesions. Top 5 pathways most commonly increased or decreased across populations were identified to ascertain potential niche-wide changes associated with aggressive lesions. 
A

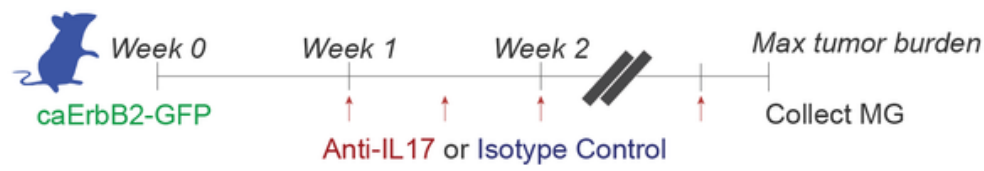

C

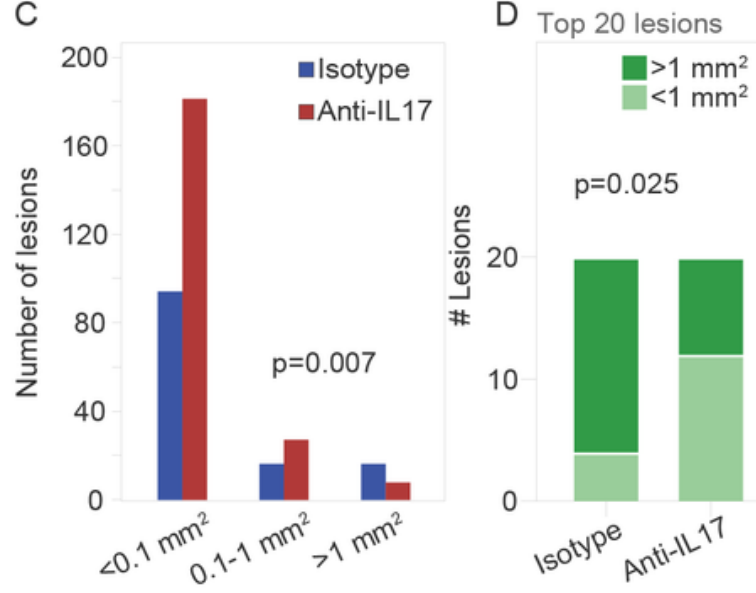

\section{E}

B

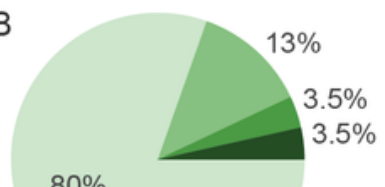

$80 \%$

$<0.1 \mathrm{~mm}^{2}$ $0.1-1 \mathrm{~mm}^{2}$ $1-10 \mathrm{~mm}^{2}$

F - $>10 \mathrm{~mm}^{2}$

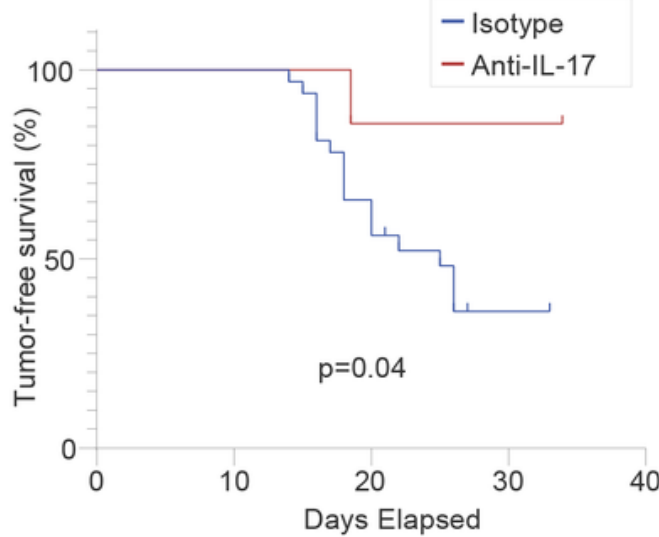

G

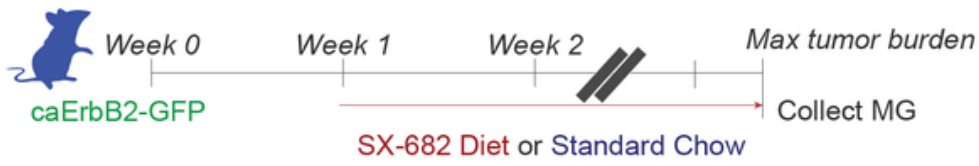

I

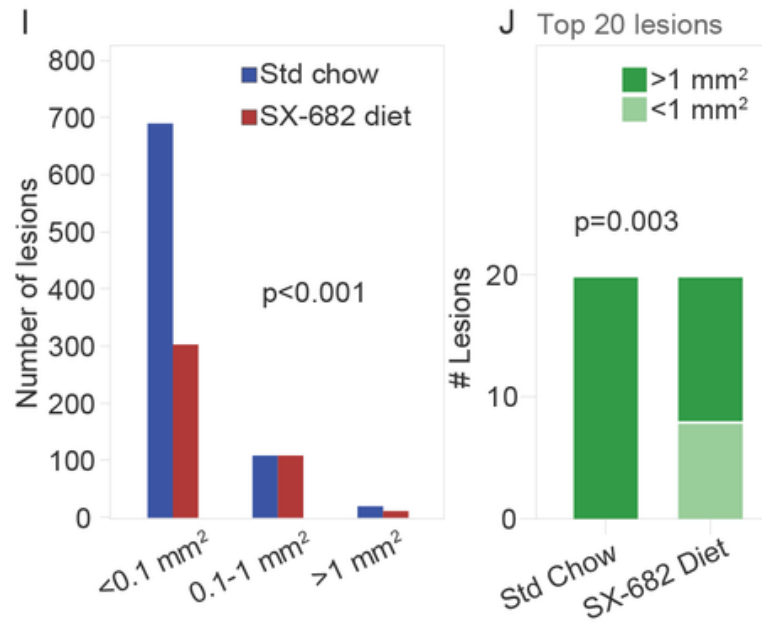

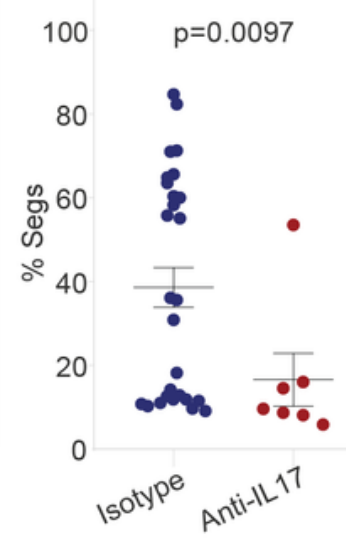

$\mathrm{H}$
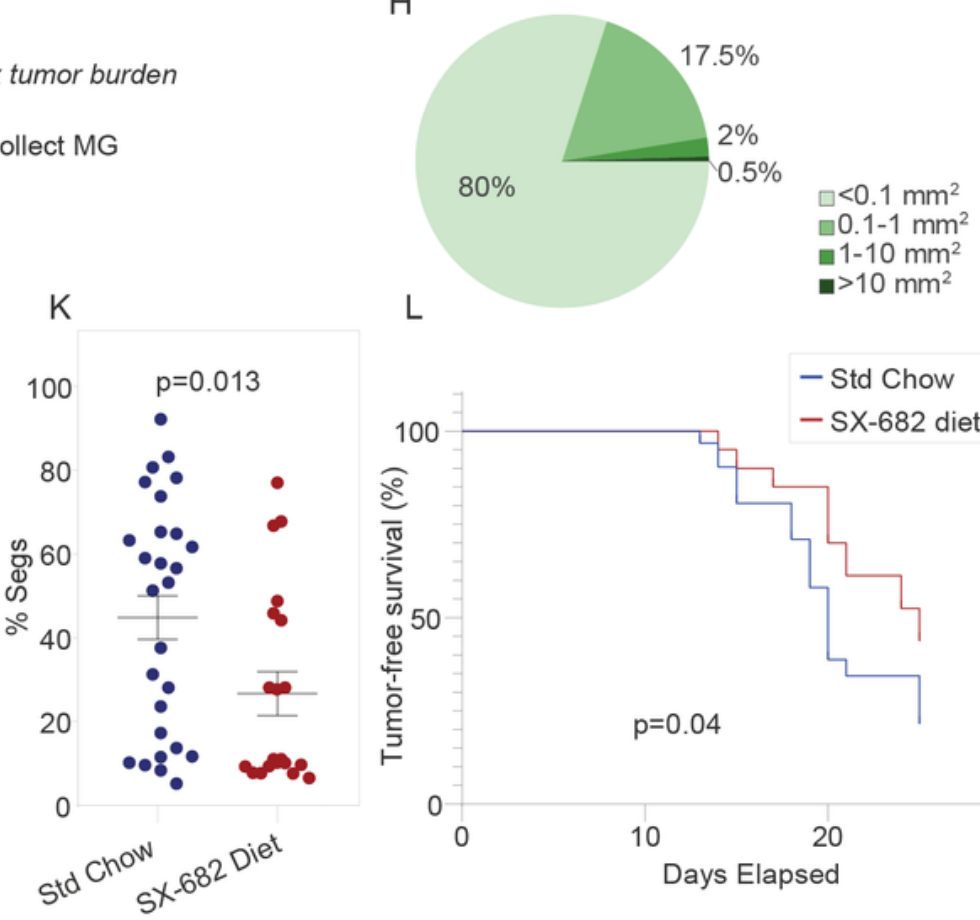

L

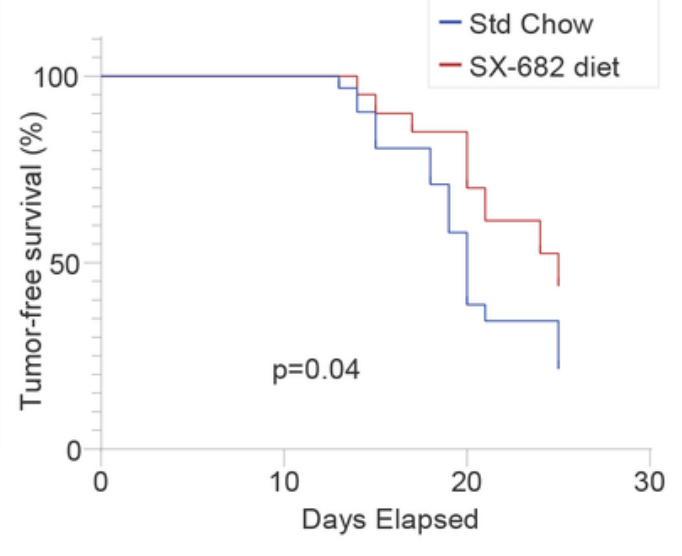

\section{Figure 7}

Inhibition of IL-17 and granulocyte recruitment reduces aggressive niche formation and delays tumor progression Animals were treated with anti-IL-17/17RA or isotype control (A-F), and SX-682 diet or standard chow (G-L). (A, G) Schema showing experimental timeline: animals were allowed to form lesions for one week then treated until endpoint. All animals were collected when any one animal reached maximum tumor burden. Quantification of lesion area from animals treated with anti-IL-17/17RA or 
isotype control (B-D), treated in the same cohort, and SX-682 diet or standard chow (H-J), treated in the same cohort. Only mammary glands bearing lesions could be quantified. Anti-IL-17 study, $\mathrm{n}=5$ per group; SX-682 study, $n=8$ per group. (B, H) Pie chart showing proportion of lesions by size for Anti-IL-17 study (B) and SX-682 study $(H)$. (C, I) Quantification of lesion area and number binned by size, with p-value calculated by Pearson's Chi-squared test, for Anti-IL-17 study (Chi-squared $=10.014, \mathrm{df}=2)(\mathrm{C}$ ) and SX682 study (Chi-squared $=29.462, \mathrm{df}=2)(I) .(D, J)$ Stacked column graph of 20 largest lesions per group binned by size for Anti-IL-17 study (D) and SX-682 study (J). P-value calculated by two-sided Fisher's exact test. $(E, K)$ Percent segmented cells measured by complete blood count from peripheral blood collected at time of euthanasia and mammary gland collection. P-value was calculated using two-sided Wilcoxon rank sum test; error bars indicate standard error of the mean (S.E.M.). Each dot represents one animal. (E) Anti-IL-17 group, $\mathrm{n}=7$; control group, $\mathrm{n}=32$ pooled from multiple identically-treated control cohorts; $U=43$. (K) SX-682 group, $n=20$; control group, $n=29$ pooled from multiple identically-treated cohorts, $\mathrm{U}=169.5$. $(\mathrm{F}, \mathrm{L})$ Kaplan-Meier plot showing tumor-free survival curves of treated animals. P-value calculated using log-rank (Mantel-Cox) test. (F) Anti-IL-17 group, $n=7$; control group, $n=32$ pooled from multiple identically-treated cohorts. (L) SX-682 group, $n=20$; control group, $n=29$ pooled from multiple identically-treated cohorts.

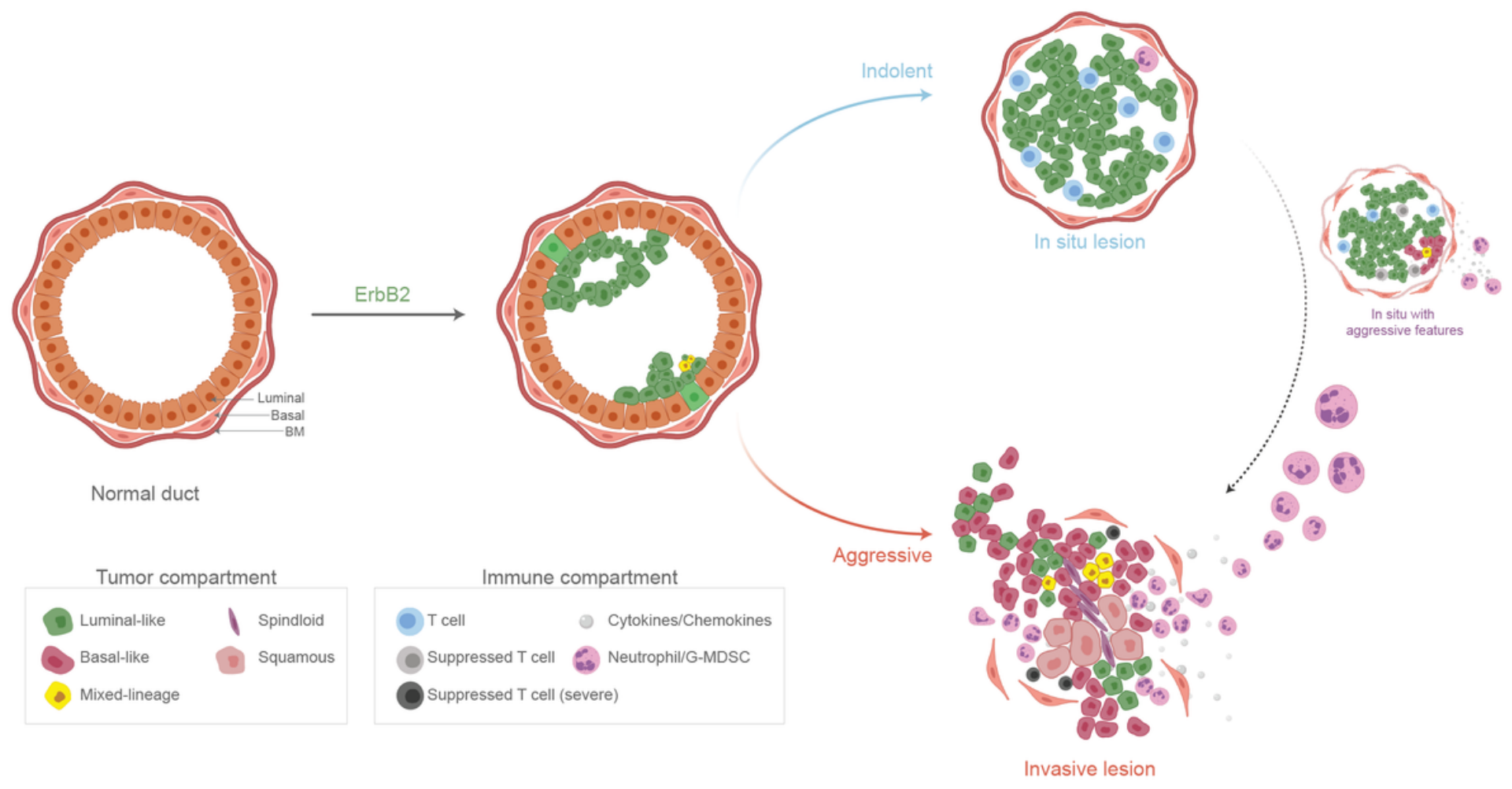

\section{Figure 8}

Indolent and aggressive lesions exhibit divergent tumor-immune niches Following introduction of the caErbB2 oncogene, transformed mammary epithelial cells form very small, early-stage lesions within mammary ducts. Over time, lesions diverge to form either indolent lesions or aggressive lesions. Indolent lesions are characterized by stereotypic, luminal-like populations, and immune infiltration into indolent lesions is limited and tend to be non-suppressed lymphoid (T cells) rather than myeloid. In contrast, 
aggressive lesions exhibit heterogeneous and functionally divergent tumor populations, including expanded basal-like populations as well as metaplastic and mixed-lineage tumor cells. Immune infiltration into an IL-17-active niche is heavy, primarily comprised of neutrophils or granulocytic myeloidderived suppressor cells (G-MDSCs), while T cells are diminished in both number and activity. Indolent lesions may transition to become aggressive, evidenced by intermediate lesions that appear indolent but exhibit sub-niche expansion of basal-like cells and recruit granulocytes that are normally restricted to aggressive lesions. BM - basement membrane

\section{Supplementary Files}

This is a list of supplementary files associated with this preprint. Click to download.

- Sinha2021Supplementarylnformation.pdf 